\title{
Global gradient estimates for degenerate parabolic equations in nonsmooth domains
}

\author{
Mikko Parviainen, \\ Helsinki University of Technology \\ Institute of Mathematics \\ P.O.Box 1100 \\ FI-02015 TKK \\ Finland \\ Mikko.Parviainen@tkk.fi \\ 7.11.2007
}

\begin{abstract}
This paper studies the global regularity theory for degenerate nonlinear parabolic partial differential equations. Our objective is to show that weak solutions belong to a higher Sobolev space than assumed a priori if the complement of the domain satisfies a capacity density condition and if the boundary values are sufficiently smooth. Moreover, we derive integrability estimates for the gradient. The results extend to the parabolic systems as well. The higher integrability estimates provide a useful tool in several applications.
\end{abstract}

\section{INTRODUCTION}

Higher integrability questions have been extensively studied over the last few decades. In this paper, we investigate the parabolic equations of the type

$$
\frac{\partial u}{\partial t}=\operatorname{div} A(x, t, \nabla u)
$$

where $A(x, t, \nabla u)$ satisfies the well-known Carathéodory-type conditions and the $p$-growth conditions. In particular, the results apply to the parabolic $p$-Laplace equation

with $2 \leq p<\infty$.

$$
\frac{\partial u}{\partial t}=\operatorname{div}\left(|\nabla u|^{p-2} \nabla u\right)
$$

Weak solutions of the above equations locally belong to a slightly higher Sobolev space than assumed a priori, as Kinnunen and Lewis proved in [19]. We intend to show that this also holds globally, that is, up to the boundary. To this end, we prove in Theorem 4.7 that the gradient of a weak solution satisfies a global reverse Hölder inequality. In contrast to the local case, the regularity of the boundary, as well as the boundary and initial values, play a role in the proofs. We assume that the complement of the domain satisfies a capacity density condition, which is essentially sharp for our main results. In addition, the boundary values are assumed to belong to an appropriate higher Sobolev space. Note, however, that the results of this paper are already nontrivial for regular domains and smooth boundary values.

The proofs are based on Caccioppoli and Sobolev-Poincaré-type inequalities, as well as on the self-improving property of a reverse Hölder inequality. Due to

2000 Mathematics Subject Classification. 35K60, 35K55, 35K15, 49N60.

Key words and phrases. boundary value problem, Gehring lemma, global higher integrability, initial value problem, reverse Hölder inequality. 
nonquadratic growth conditions, the proofs apply intrinsic scaling and covering arguments. One of the advantages of this method lies in the fact that it can be employed to a wide variety of problems. Indeed, the proofs extend to parabolic systems of the form

$$
\frac{\partial u_{i}}{\partial t}=\operatorname{div} A_{i}(x, t, \nabla u), \quad i=1,2, \ldots, N,
$$

although we consider the scalar case for simplicity.

Motivation for studying the higher integrability comes from applications to partial regularity (see, for example, Giaquinta-Modica [13]) and stability questions, to mention a few. On the other hand, the regularity properties of solutions are often interesting in their own right.

The first higher integrability results apparently date back to a 1957 paper of Bojarski, [5]. Later, Elcrat and Meyers proved the local higher integrability for nonlinear elliptic systems in [9] (see also the monograph of Giaquinta [12]). In [14], Giaquinta and Struwe studied the similar questions for systems of parabolic equations with quadratic growth conditions. In addition, Arkhipova has considered the global reqularity questions for parabolic systems, for example, in [3] and [4]. For recent higher integrability results, see Acerbi-Mingione [1]. See also DuzaarMingione [8] for further parabolic regularity results and [7] for the corresponding results of $p$-Laplacean type.

In [15], Granlund showed that an elliptic minimizer has the higher integrability property if the complement of the domain satisfies a measure density condition. Later, Kilpeläinen and Koskela generalized the elliptic results to the uniform capacity density condition in [18]. For a good survey of the boundary regularity, see Section 8 of Mikkonen [24]. Recently, it was shown in Parviainen [25] that parabolic quasiminimizers with quadratic growth conditions have a global higher integrability property.

\section{Preliminaries}

Let $\Omega$ be a bounded open set in $\mathbb{R}^{n}, n \geq 2$ and let $p \geq 2$. We study the equation

$$
\frac{\partial u}{\partial t}=\operatorname{div} A(x, t, \nabla u), \quad(x, t) \in \Omega \times(0, T),
$$

where $u: \Omega \times(0, T) \rightarrow \mathbb{R}, A: \Omega \times(0, T) \times \mathbb{R}^{n} \rightarrow \mathbb{R}^{n}$, and $A$ satisfies the following conditions.

(1) $(x, t) \mapsto A(x, t, \xi)$ is measurable for every $\xi$,

(2) $\xi \mapsto A(x, t, \xi)$ is continuous for almost every $(x, t)$,

(3) there exist constants $0<\alpha \leq \beta<\infty$ such that for every $\xi$ and for almost every $(x, t)$, we have $A(x, t, \xi) \cdot \xi \geq \alpha|\xi|^{p}$ and $|A(x, t, \xi)| \leq \beta|\xi|^{p-1}$.

As usual, $W^{1, p}(\Omega)$ denotes the Sobolev space of functions in $L^{p}(\Omega)$ whose first distributional partial derivatives belong to $L^{p}(\Omega)$ with the norm

$$
\|u\|_{W^{1, p}(\Omega)}=\|u\|_{L^{p}(\Omega)}+\|\nabla u\|_{L^{p}(\Omega)} .
$$

The Sobolev space $W_{0}^{1, p}(\Omega)$ is a completion of $C_{0}^{\infty}(\Omega)$ in the norm of $W^{1, p}(\Omega)$.

The parabolic space $L^{p}\left(0, T ; W^{1, p}(\Omega)\right)$ is a collection of measurable functions $u(x, t)$ such that for almost every $t \in(0, T)$, the function $x \mapsto u(x, t)$ belongs to 
$W^{1, p}(\Omega)$ and the norm

$$
\|u\|_{L^{p}\left(0, T ; W^{1, p}(\Omega)\right)}=\left(\int_{0}^{T}\|u\|_{W^{1, p}(\Omega)}^{p} \mathrm{~d} t\right)^{1 / p}
$$

is finite. Analogously, the space $L^{p}\left(0, T ; W_{0}^{1, p}(\Omega)\right)$ is a collection of measurable functions $u(x, t)$ such that for almost every $t \in(0, T)$, the function $x \mapsto u(x, t)$ belongs to $W_{0}^{1, p}(\Omega)$ and

$$
\|u\|_{L^{p}\left(0, T ; W^{1, p}(\Omega)\right)}<\infty
$$

The parabolic Sobolev space $W^{1,2}\left(0, T ; L^{2}(\Omega)\right)$ is defined as

$$
\begin{aligned}
& W^{1,2}\left(0, T ; L^{2}(\Omega)\right) \\
& \quad=\left\{\varphi \in L^{2}\left(0, T ; L^{2}(\Omega)\right): \frac{\partial \varphi}{\partial t} \in L^{2}\left(0, T ; L^{2}(\Omega)\right)\right\}
\end{aligned}
$$

with the norm

$$
\|\varphi\|_{W^{1,2}\left(0, T ; L^{2}(\Omega)\right)}=\|\varphi\|_{L^{2}\left(0, T ; L^{2}(\Omega)\right)}+\left\|\frac{\partial \varphi}{\partial t}\right\|_{L^{2}\left(0, T ; L^{2}(\Omega)\right)} .
$$

Finally, the space $C\left([0, T] ; L^{2}(\Omega)\right)$ comprises all continuous functions $u:[0, T] \rightarrow$ $L^{2}(\Omega)$ (that is, $u$ is continuous with respect to $t$ in the norm $\|\cdot\|_{L^{2}(\Omega)}$ ) such that

$$
\|u\|_{C\left([0, T] ; L^{2}(\Omega)\right)}=\max _{t \in[0, T]}\|u(\cdot, t)\|_{L^{2}(\Omega)}<\infty .
$$

A function $u$ belonging to the space $L_{\mathrm{loc}}^{p}\left(0, T ; W_{\mathrm{loc}}^{1, p}(\Omega)\right)$ is a weak solution to (2.1) if

$$
-\int_{0}^{T} \int_{\Omega} u \frac{\partial \phi}{\partial t} \mathrm{~d} x \mathrm{~d} t+\int_{0}^{T} \int_{\Omega} A(x, t, \nabla u) \cdot \nabla \phi \mathrm{d} x \mathrm{~d} t=0,
$$

for every $\phi \in C_{0}^{\infty}(\Omega \times(0, T))$.

There is a well-recognized difficulty in proving Caccioppoli-type estimates for weak solutions: One often needs a test function depending on $u$ itself, but $u$ may not be admissible. For example, the time derivative of the test function contains $\frac{\partial u}{\partial t}$, which does not necessarily exist as a function. There are several ways to treat this difficulty: We may, for example, use the Steklov averages, as on page 25 in DiBenedetto's monograph [6], or we may use the standard mollifications. Here we adopt the latter approach and set

$$
\tilde{\phi}(x, t)=\int_{\mathbb{R}} \phi(x, t-s) \zeta_{\varepsilon}(s) \mathrm{d} s,
$$

where $\phi \in C_{0}^{\infty}(\Omega \times(0, T))$ and $\zeta_{\varepsilon}(s)$ is a standard mollifier, whose support is contained in $(-\varepsilon, \varepsilon)$ with $\varepsilon<\operatorname{dist}(\operatorname{spt}(\phi), \Omega \times\{0, T\})$. We insert $\tilde{\phi}$ into $(2.2)$, change variables and apply Fubini's theorem to obtain

$$
-\int_{0}^{T} \int_{\Omega} u_{\varepsilon} \frac{\partial \phi}{\partial t} \mathrm{~d} z+\int_{0}^{T} \int_{\Omega} A(x, t, \nabla u)_{\varepsilon} \cdot \nabla \phi \mathrm{d} z=0 .
$$

Here $u_{\varepsilon}$ and $A(x, t, \nabla u)_{\varepsilon}$ denote the standard mollifications in the time direction.

We finish this section with notation used throughout the work. Let

$$
D=\Omega \times(0, T)
$$


be a space-time cylinder. We denote the points of the cylinder by $z=(x, t)$ and employ a shorthand notation $\mathrm{d} z=\mathrm{d} x \mathrm{~d} t$. Let $z_{0}=\left(x_{0}, t_{0}\right) \in D$ and $\theta, \rho>0$. Then we denote

$$
\begin{aligned}
& B_{\rho}\left(x_{0}\right)=\left\{x \in \mathbb{R}^{n}:\left|x-x_{0}\right|<\rho\right\}, \\
& \bar{B}_{\rho}\left(x_{0}\right)=\left\{x \in \mathbb{R}^{n}:\left|x-x_{0}\right| \leq \rho\right\}
\end{aligned}
$$

and

$$
\Lambda_{\theta \rho^{2}}\left(t_{0}\right)=\left(t_{0}-\frac{1}{2} \theta \rho^{2}, t_{0}+\frac{1}{2} \theta \rho^{2}\right) .
$$

Further, a space-time cylinder in $\mathbb{R}^{n+1}$ is denoted by

$$
Q_{\rho, \theta \rho^{2}}\left(z_{0}\right)=Q_{\rho, \theta \rho^{2}}\left(x_{0}, t_{0}\right)=B_{\rho}\left(x_{0}\right) \times \Lambda_{\theta \rho^{2}}\left(t_{0}\right) .
$$

When no confusion arises, we shall omit the reference points and simply write $B_{\rho}$, $\Lambda_{\theta \rho^{2}}$ and $Q_{\rho, \theta \rho^{2}}$. The integral average of $u$ is denoted by

$$
u_{\rho}(t)=f_{B_{\rho}} u(x, t) \mathrm{d} x=\frac{1}{\left|B_{\rho}\right|} \int_{B_{\rho}} u(x, t) \mathrm{d} x,
$$

where $\left|B_{\rho}\right|$ denotes the Lebesgue measure of $B_{\rho}$. Finally, $\phi^{\prime}$ sometimes denotes the time derivative of $\phi$ instead of $\frac{\partial \phi}{\partial t}$.

\section{Estimates NEAR the Boundary}

In this section, we derive estimates near the lateral boundary $\partial \Omega \times(0, T)$. These estimates are applied in Section 4 in order to prove a reverse Hölder inequality.

A Lebesgue-type initial condition and a Sobolev-type boundary condition turn out to be convenient for our purposes. To be more specific, we say that $u$ is a global solution if $u \in L^{p}\left(0, T ; W^{1, p}(\Omega)\right)$ satisfies $(2.2)$ as well as the initial and boundary conditions:

$$
\begin{aligned}
& u(\cdot, t)-\varphi(\cdot, t) \in W_{0}^{1, p}(\Omega) \quad \text { for almost every } t \in(0, T) \\
& \text { and } \\
& \frac{1}{h} \int_{0}^{h} \int_{\Omega}|u-\varphi|^{2} \mathrm{~d} x \mathrm{~d} t \rightarrow 0 \quad \text { as } \quad h \rightarrow 0,
\end{aligned}
$$

for a given

$$
\varphi \in W^{1,2}\left(0, T ; L^{2}(\Omega)\right) \cap L^{p}\left(0, T ; W^{1, p}(\Omega)\right) .
$$

Observe that already smooth $\varphi$ leads to a nontrivial theory. We start with a Caccioppoli-type inequality.

Lemma 3.2 (CACCIOPPOLI). Let $u$ be a global solution with the boundary and initial conditions (3.1). Let $\theta>0$, suppose that $0<\rho<M$ for some $M>0$, and let $Q_{\rho, \theta \rho^{2}}=Q_{\rho, \theta \rho^{2}}\left(x_{0}, t_{0}\right) \subset \mathbb{R}^{n+1}$. Then there exists a constant $c=c(n, p, M, \alpha, \beta)>$ 0 such that

$$
\begin{aligned}
& \int_{Q_{\rho, \theta \rho^{2}} \cap D}|\nabla u|^{p} \mathrm{~d} z+\operatorname{essup}_{t \in \Lambda_{\theta \rho^{2}} \cap(0, T)} \int_{B_{\rho} \cap \Omega}|u-\varphi|^{2} \mathrm{~d} x \\
& \leq \frac{c}{\theta \rho^{2}} \int_{Q_{4 \rho, \theta(4 \rho) 2} \cap D}|u-\varphi|^{2} \mathrm{~d} z+\frac{c}{\rho^{p}} \int_{Q_{4 \rho, \theta(4 \rho)^{2}} \cap D}|u-\varphi|^{p} \mathrm{~d} z \\
&+c \int_{Q_{4 \rho, \theta(4 \rho))^{2}} \cap D} f^{p} \mathrm{~d} z
\end{aligned}
$$

where $D=\Omega \times(0, T)$ and $f=\left(\left|\varphi^{\prime}\right|^{p /(p-1)}+|\nabla \varphi|^{p}\right)^{1 / p}$. 
Proof: We may assume that $Q_{\rho, \theta \rho^{2}} \cap D \neq \emptyset$ since otherwise the claim is trivial. Let $t_{1} \in \Lambda_{\theta \rho^{2}} \cap(0, T)$. We define $\chi_{0, t_{1}}^{h}(t)$ to be a piecewise linear approximation of a characteristic function such that

$$
\begin{gathered}
\chi_{0, t_{1}}^{h}(t)=1 \quad \text { as } h \leq t \leq t_{1}-h, \\
\chi_{0, t_{1}}^{h}(t)=0 \quad \text { as } \quad t \leq h / 10 \quad \text { or } \quad t \geq t_{1}-h / 10,
\end{gathered}
$$

and

$$
\left|\left(\chi_{0, t_{1}}^{h}(t)\right)^{\prime}\right| \leq \frac{10}{9 h}
$$

Further, denote by $\chi_{0, t_{1}}^{h, \varepsilon}(t), u_{\varepsilon}$ and $\varphi_{\varepsilon}$ the standard mollifications in the time direction for $\varepsilon<h / 20$. We choose a test function

$$
\phi_{\varepsilon}(x, t)=\eta^{p}(x, t)\left(u_{\varepsilon}(x, t)-\varphi_{\varepsilon}(x, t)\right) \chi_{0, t_{1}}^{h, \varepsilon}(t),
$$

where $\eta \in C_{0}^{\infty}\left(\mathbb{R}^{n+1}\right)$ is a cut-off function such that spt $\eta \subset Q_{4 \rho, \theta(4 \rho)^{2}}, \eta(x, t)=1$ in $Q_{\rho, \theta \rho^{2}}, 0 \leq \eta \leq 1$, and

$$
\rho|\nabla \eta|+\theta \rho^{2}\left|\frac{\partial \eta}{\partial t}\right| \leq c .
$$

The mollification in the time direction does not affect the lateral boundary values, and thus $\phi_{\varepsilon}(\cdot, t) \in W_{0}^{1, p}(\Omega)$ for almost every $t \in(0, T)$.

To begin with, we insert the test function into (2.3) and manipulate the first term to have

$$
-\int_{D} u_{\varepsilon} \phi_{\varepsilon}^{\prime} \mathrm{d} z=-\int_{D}\left(u_{\varepsilon}-\varphi_{\varepsilon}\right) \phi_{\varepsilon}^{\prime} \mathrm{d} z-\int_{D} \varphi_{\varepsilon} \phi_{\varepsilon}^{\prime} \mathrm{d} z .
$$

By integrating the first term on the right hand side of (3.4) by parts, we obtain

$$
\begin{aligned}
& -\int_{D}\left(u_{\varepsilon}-\varphi_{\varepsilon}\right) \phi_{\varepsilon}^{\prime} \mathrm{d} z \\
& \quad=-\int_{D}\left(\left(u_{\varepsilon}-\varphi_{\varepsilon}\right)^{2}\left(\eta^{p} \chi_{0, t_{1}}^{h, \varepsilon}\right)^{\prime} \mathrm{d} z+\frac{1}{2}\left[\left(u_{\varepsilon}-\varphi_{\varepsilon}\right)^{2}\right]^{\prime} \eta^{p} \chi_{0, t_{1}}^{h, \varepsilon}\right) \mathrm{d} z \\
& =-\frac{1}{2} \int_{D}\left(u_{\varepsilon}-\varphi_{\varepsilon}\right)^{2}\left(\eta^{p} \chi_{0, t_{1}}^{h, \varepsilon}\right)^{\prime} \mathrm{d} z .
\end{aligned}
$$

As a next step, we take limits, apply the initial condition, and use the well-known convergence properties of mollified functions. We deduce for almost every $t_{1} \in$ $\Lambda_{\theta \rho^{2}} \cap(0, T)$ that

$$
\begin{aligned}
-\int_{D}\left(u_{\varepsilon}-\varphi_{\varepsilon}\right) \phi_{\varepsilon}^{\prime} \mathrm{d} z \rightarrow & -\frac{1}{2} \int_{\Omega \times\left(0, t_{1}\right)}|u-\varphi|^{2} p \eta^{p-1} \eta^{\prime} \mathrm{d} z \\
& +\frac{1}{2} \int_{\Omega}\left|u\left(x, t_{1}\right)-\varphi\left(x, t_{1}\right)\right|^{2} \eta^{p}\left(x, t_{1}\right) \mathrm{d} x
\end{aligned}
$$

as first $\varepsilon \rightarrow 0$ and then $h \rightarrow 0$. Because we take the limits in this order, the mollifications are well defined. Observe also that the initial boundary term disappears at $t=0$ because of the initial condition. 
Then we combine the previous estimates, integrate the last term of (3.4) by parts, and obtain

$$
\begin{aligned}
-\int_{D} u_{\varepsilon} \phi_{\varepsilon}^{\prime} \mathrm{d} z \rightarrow & -\frac{1}{2} \int_{\Omega \times\left(0, t_{1}\right)}|u-\varphi|^{2} p \eta^{p-1} \eta^{\prime} \mathrm{d} z \\
& +\frac{1}{2} \int_{\Omega}\left(u\left(x, t_{1}\right)-\varphi\left(x, t_{1}\right)\right)^{2} \eta^{p}\left(x, t_{1}\right) \mathrm{d} x \\
& +\int_{\Omega \times\left(0, t_{1}\right)} \varphi^{\prime} \eta^{p}(u-\varphi) \mathrm{d} z
\end{aligned}
$$

as first $\varepsilon \rightarrow 0$ and then $h \rightarrow 0$.

Inserting the test function into the second term of (2.3) implies

$$
\begin{aligned}
& \int_{D} A(x, t, \nabla u)_{\varepsilon} \cdot \nabla\left(\eta^{p}\left(u_{\varepsilon}-\varphi_{\varepsilon}\right) \chi_{0, t_{1}}^{h, \varepsilon}\right) \mathrm{d} z \\
& \quad \rightarrow \int_{\Omega \times\left(0, t_{1}\right)} A(x, t, \nabla u) \cdot\left[p \eta^{p-1} \nabla \eta(u-\varphi)+\eta^{p}(\nabla u-\nabla \varphi)\right] \mathrm{d} z
\end{aligned}
$$

as first $\varepsilon \rightarrow 0$ and then $h \rightarrow 0$.

Collecting the facts, we arrive at

$$
\begin{aligned}
& \int_{\Omega \times\left(0, t_{1}\right)} \eta^{p} A(x, t, \nabla u) \cdot \nabla u \mathrm{~d} z+\frac{1}{2} \int_{\Omega}\left|u\left(x, t_{1}\right)-\varphi\left(x, t_{1}\right)\right|^{2} \eta^{p}\left(x, t_{1}\right) \mathrm{d} x \\
& \leq \frac{1}{2} \int_{\Omega \times\left(0, t_{1}\right)}|u-\varphi|^{2} p \eta^{p-1}\left|\eta^{\prime}\right| \mathrm{d} z+\int_{\Omega \times\left(0, t_{1}\right)}\left|\varphi^{\prime}\right| \eta^{p}|u-\varphi| \mathrm{d} z \\
& \quad+\int_{\Omega \times\left(0, t_{1}\right)}|A(x, t, \nabla u)| p \eta^{p-1}|\nabla \eta||u-\varphi| \mathrm{d} z \\
& \quad+\int_{\Omega \times\left(0, t_{1}\right)}|A(x, t, \nabla u)| \eta^{p}|\nabla \varphi| \mathrm{d} z
\end{aligned}
$$

In view of our hypotheses on $A$, the first term on the left hand side satisfies the inequality

$$
\alpha \int_{\Omega \times\left(0, t_{1}\right)} \eta^{p}|\nabla u|^{p} \mathrm{~d} z \leq \int_{\Omega \times\left(0, t_{1}\right)} \eta^{p} A(x, t, \nabla u) \cdot \nabla u \mathrm{~d} z .
$$

Since $\rho<M$, there exists a constant $c>0$ such that $1 \leq c / \rho^{p}$, where $c$, of course, depends on $M$. Consequently, Young's inequality implies

$$
\begin{aligned}
\int_{\Omega \times\left(0, t_{1}\right)} & \left|\varphi^{\prime}\right| \eta^{p}|u-\varphi| \mathrm{d} z \\
& \leq \varepsilon \int_{\Omega \times\left(0, t_{1}\right)}\left|\varphi^{\prime}\right|^{p /(p-1)} \eta^{p} \mathrm{~d} z+\frac{c}{\rho^{p}} \int_{\Omega \times\left(0, t_{1}\right)}|u-\varphi|^{p} \eta^{p} \mathrm{~d} z,
\end{aligned}
$$

where the constant depends on $M$ and $\varepsilon>0$. Next we estimate the third term on the right hand side of (3.5). Young's inequality and the structural assumptions on $A$ lead to

$$
\begin{aligned}
\int_{\Omega \times\left(0, t_{1}\right)} & |A(x, t, \nabla u)| p \eta^{p-1}|\nabla \eta||u-\varphi| \mathrm{d} z \\
& \leq \varepsilon \int_{\Omega \times\left(0, t_{1}\right)}|\nabla u|^{p} \eta^{p} \mathrm{~d} z+c \int_{\Omega \times\left(0, t_{1}\right)}|\nabla \eta|^{p}|u-\varphi|^{p} \mathrm{~d} z .
\end{aligned}
$$


A similar reasoning allows us to estimate the fourth term on the right hand side of (3.5) as

$$
\begin{aligned}
\int_{\Omega \times\left(0, t_{1}\right)} & |A(x, t, \nabla u)| \eta^{p}|\nabla \varphi| \mathrm{d} z \\
& \leq \varepsilon \int_{\Omega \times\left(0, t_{1}\right)}|\nabla u|^{p} \eta^{p} \mathrm{~d} z+c \int_{\Omega \times\left(0, t_{1}\right)} \eta^{p}|\nabla \varphi|^{p} \mathrm{~d} z .
\end{aligned}
$$

Let us then estimate the second term on the left hand side of (3.5). We can choose $t_{1} \in \Lambda_{\theta \rho^{2}} \cap(0, T)$ such that

$$
\begin{aligned}
& \frac{1}{2} \underset{t \in \Lambda_{\theta \rho^{2}} \cap(0, T)}{\operatorname{ess} \sup _{B_{\rho} \cap \Omega}} \int|u-\varphi|^{2} \eta^{p} \mathrm{~d} x \\
& \quad \leq \int_{\Omega}\left|u\left(x, t_{1}\right)-\varphi\left(x, t_{1}\right)\right|^{2} \eta^{p}\left(x, t_{1}\right) \mathrm{d} x
\end{aligned}
$$

Finally, we combine the above estimates with (3.5) and choose $\varepsilon>0$ small enough to absorb

$$
\varepsilon \int_{\Omega \times\left(0, t_{1}\right)} \eta^{p}|\nabla u|^{p} \mathrm{~d} z
$$

into the left hand side. Since $\eta$ satisfies condition (3.3), we obtain the claim.

The regularity of the boundary plays a role in the global higher integrability. In this paper, we assume that the complement of the domain satisfies a uniform capacity density condition.

Let $1<p<\infty$. The variational $p$-capacity of a compact set $C \subset \Omega$ is defined to be

$$
\operatorname{cap}_{p}(C, \Omega)=\inf _{g} \int_{\Omega}|\nabla g|^{p} \mathrm{~d} x,
$$

where the infimum is taken over all the functions $g \in C_{0}^{\infty}(\Omega)$ such that $g=1$ in $C$. To define the variational $p$-capacity of an open set $U \subset \Omega$, we take the supremum over the capacities of the compact sets belonging to $U$. The variational $p$-capacity of an arbitrary set $E \subset \Omega$ is defined by taking the infimum over the capacities of the open sets containing $E$. For further details, see Chapter 4 of Evans-Gariepy [10], Chapter 2 of Heinonen-Kilpeläinen-Martio [17], or Chapter 2 of Malý-Ziemer $[22]$.

A set $E \subset \mathbb{R}^{n}$ is said to be of $p$-capacity zero if

$$
\operatorname{cap}_{p}(E \cap U, U)=0
$$

for all open $U \subset \mathbb{R}^{n}$. For the capacity of a ball, we obtain the following simple formula

$$
\operatorname{cap}_{p}\left(\bar{B}_{\rho}, B_{2 \rho}\right)=c \rho^{n-p},
$$

where $c>0$ depends only on $n$ and $p$.

Let us now introduce the capacity density condition which we later impose on the complement of the domain. For the higher integrability results, this condition is essentially sharp as pointed out in Remark 3.3. of Kilpeläinen-Koskela [18] in the elliptic case.

Definition 3.7. A set $E \subset \mathbb{R}^{n}$ is uniformly $p$-thick if there exist constants $\mu, \rho_{0}>0$ such that

$$
\operatorname{cap}_{p}\left(E \cap \bar{B}_{\rho}(x), B_{2 \rho}(x)\right) \geq \mu \operatorname{cap}_{p}\left(\bar{B}_{\rho}(x), B_{2 \rho}(x)\right),
$$


for all $x \in E$ and for all $0<\rho<\rho_{0}$.

If we replace the capacity with the Lebesgue measure in the definition above, then we obtain a measure density condition. A set $E$, satisfying the measure density condition, is uniformly $p$-thick for all $p>1$. If $p>n$, then every nonempty set is uniformly $p$-thick. The following lemma extends the capacity estimate in Definition 3.7.

Lemma 3.8. Let $\Omega$ be a bounded open set, and suppose that $\mathbb{R}^{n} \backslash \Omega$ is uniformly p-thick. Choose $y \in \Omega$ such that $B_{\frac{4}{3} \rho}(y) \backslash \Omega \neq \emptyset$. Then there exists a constant $\tilde{\mu}=\tilde{\mu}\left(\mu, \rho_{0}, n, p\right)>0$ such that

$$
\operatorname{cap}_{p}\left(\bar{B}_{2 \rho}(y) \backslash \Omega, B_{4 \rho}(y)\right) \geq \tilde{\mu} \operatorname{cap}_{p}\left(\bar{B}_{2 \rho}(y), B_{4 \rho}(y)\right) .
$$

Proof: Since $B_{\frac{4}{3} \rho}(y) \backslash \Omega \neq \emptyset$, we may choose $x \in \mathbb{R}^{n} \backslash \Omega$ such that $\operatorname{dist}(x, y)<\frac{4}{3} \rho$. Then

$$
B_{4 \rho}(y) \subset B_{\left(\frac{4}{3}+4\right) \rho}(x) \quad \text { and } \quad B_{\frac{2}{3} \rho}(x) \subset B_{2 \rho}(y),
$$

and hence, due to the properties of the capacity, we obtain

$$
\begin{aligned}
\operatorname{cap}_{p}\left(\bar{B}_{2 \rho}(y) \backslash \Omega, B_{4 \rho}(y)\right) & \geq \operatorname{cap}_{p}\left(\bar{B}_{2 \rho}(y) \backslash \Omega, B_{\left(\frac{4}{3}+4\right) \rho}(x)\right) \\
& \geq \operatorname{cap}_{p}\left(\bar{B}_{\frac{2}{3} \rho}(x) \backslash \Omega, B_{\left(\frac{4}{3}+4\right) \rho}(x)\right) .
\end{aligned}
$$

Lemma 2.16 of [17] provides the estimate

$$
\operatorname{cap}_{p}\left(\bar{B}_{\frac{2}{3} \rho}(x) \backslash \Omega, B_{\left(\frac{4}{3}+4\right) \rho}(x)\right) \geq c \operatorname{cap}_{p}\left(\bar{B}_{\frac{2}{3} \rho}(x) \backslash \Omega, B_{\frac{4}{3} \rho}(x)\right) .
$$

Since $\Omega$ is bounded, the uniform $p$-thickness condition holds for every $\rho$ and thus

$$
\operatorname{cap}_{p}\left(\bar{B}_{\frac{2}{3} \rho}(x) \backslash \Omega, B_{\left(\frac{4}{3}+4\right) \rho}(x)\right) \geq c \operatorname{cap}_{p}\left(\bar{B}_{\frac{2}{3} \rho}(x), B_{\frac{4}{3} \rho}(x)\right) .
$$

According to (3.6), there exists a constant $c>0$ such that

$$
\operatorname{cap}_{p}\left(\bar{B}_{\frac{2}{3} \rho}(x), B_{\frac{4}{3} \rho}(x)\right) \geq c \operatorname{cap}_{p}\left(\bar{B}_{2 \rho}(y), B_{4 \rho}(y)\right) .
$$

A combination of (3.9), (3.10) and (3.11) implies the result.

A uniformly $p$-thick domain has a deep self-improving property. This result was shown by Lewis in [21]. See also Ancona [2] and Mikkonen [24].

Theorem 3.12. Let $1<p \leq n$. If a set $E$ is uniformly $p$-thick, then there exists a constant $q=q(n, p, \mu)$ such that $1<q<p$ for which $E$ is uniformly $q$-thick.

A uniformly $q$-thick set is also uniformly $p$-thick for all $p \geq q$. This is a simple consequence of Hölder's and Young's inequalities.

Next we formulate a well-known version of the Sobolev-type inequality. For the proof, see Hedberg [16], Chapter 10 of Maz'ja's monograph [23] or Lemma 3.1 of Kilpeläinen-Koskela [18]. Later, we combine this estimate with the boundary regularity condition and obtain a boundary version of Sobolev's inequality.

The lemma employs quasicontinuous representatives of the Sobolev functions. We call $u \in W^{1, p}(\Omega) p$-quasicontinuous if for each $\varepsilon>0$ there exists an open set $U, U \subset \Omega \subset B_{R^{\prime}}$, such that $\operatorname{cap}_{p}\left(U, B_{2 R^{\prime}}\right) \leq \varepsilon$, and the restriction of $u$ to the set $\Omega \backslash U$ is finite valued and continuous.

The $p$-quasicontinuous functions are closely related to the Sobolev space $W^{1, p}(\Omega)$ : For example, if $u \in W^{1, p}(\Omega)$, then $u$ has a $p$-quasicontinuous representative. In addition, the capacity can be written in terms of quasicontinuous representatives. 
From now on, we only consider the case $p \leq n$ for simplicity. This restriction is only technical and in this way, we avoid repeating essentially the same proofs with more complicated powers emerging from the different versions of the SobolevPoincaré inequalities.

Lemma 3.13. Suppose that $q \in(1, p)$ and that $u \in W^{1, q}\left(B_{2 \rho}\right)$ is q-quasicontinuous. Denote

$$
N_{B_{\rho}}(u)=\left\{x \in \bar{B}_{\rho}: u(x)=0\right\}
$$

and choose $\tilde{q} \in\left[q, q^{*}\right]$, where $q^{*}=q n /(n-q)$. Then there exists a constant $c=$ $c(n, q)>0$ such that

$$
\left(f_{B_{2 \rho}}|u|^{\tilde{q}} \mathrm{~d} x\right)^{1 / \tilde{q}} \leq\left(\frac{c}{\operatorname{cap}_{q}\left(N_{B_{\rho}}(u), B_{2 \rho}\right)} \int_{B_{2 \rho}}|\nabla u|^{q} \mathrm{~d} x\right)^{1 / q} .
$$

The above estimate also holds if the powers on the both sides are replaced by $p$.

Lemma 3.14. Suppose that $u \in W^{1, p}\left(B_{2 \rho}\right)$ is $p$-quasicontinuous and let $N_{B_{\rho}}(u)$ be as above. Then there exists a constant $c=c(n, p)>0$ such that

$$
\left(f_{B_{2 \rho}}|u|^{p} \mathrm{~d} x\right)^{1 / p} \leq\left(\frac{c}{\operatorname{cap}_{p}\left(N_{B_{\rho}}(u), B_{2 \rho}\right)} \int_{B_{2 \rho}}|\nabla u|^{p} \mathrm{~d} x\right)^{1 / p} .
$$

In order to derive a reverse Hölder inequality, we estimate the right hand side of Caccioppoli's inequality in terms of the gradient. A natural idea is to use Sobolev's inequality, but there is a principal difficulty in the parabolic case: We assume little regularity for a weak solution $u$ in the time direction, and Sobolev's inequality is not applicable in space-time cylinders as such. Nevertheless, weak solutions satisfy the following parabolic Sobolev's inequality.

Lemma 3.15 (PARABOLIC SOBOLEV). Let $u$ be a global solution with the boundary and initial conditions (3.1). Suppose that $\mathbb{R}^{n} \backslash \Omega$ is uniformly $p$-thick. Let $\theta>0$ and choose $Q_{\rho, \theta \rho^{2}}=Q_{\rho, \theta \rho^{2}}\left(x_{0}, t_{0}\right) \subset \mathbb{R}^{n+1}$ such that $B_{\frac{4}{3} \rho}\left(x_{0}\right) \backslash \Omega \neq \emptyset$. Further, choose $M$ such that $\rho<M$. Then there exists a constant $c=c\left(n, p, M, \mu, \rho_{0}, \alpha, \beta\right)>0$ so that

$$
\begin{aligned}
\underset{\substack{t \in \Lambda_{\theta \rho^{2}} \cap(0, T) \\
\operatorname{ess} \sup _{i \rho} \cap}}{ } \int_{B_{\rho} \cap u-\left.\varphi\right|^{2} \mathrm{~d} x} & \\
\leq & c \rho^{n+2}\left(\frac{1}{\left|Q_{4 \rho, \theta(4 \rho)^{2}}\right|} \int_{Q_{4 \rho, \theta(4 \rho)^{2}} \cap D}|\nabla(u-\varphi)|^{p} \mathrm{~d} z\right)^{2 / p} \\
& +c \int_{Q_{4 \rho, \theta(4 \rho)^{2}} \cap D}|\nabla(u-\varphi)|^{p} \mathrm{~d} z+c \int_{Q_{4 \rho, \theta(4 \rho)^{2}} \cap D} f^{p} \mathrm{~d} z
\end{aligned}
$$

where $f=\left(\left|\varphi^{\prime}\right|^{p /(p-1)}+|\nabla \varphi|^{p}\right)^{1 / p}$.

Proof: In order to prove the claim, we estimate the right hand side of Caccioppoli's inequality by applying Lemma 3.14 and the uniform capacity density condition. 
Lemma 3.2 provides the estimate

$$
\begin{aligned}
& \underset{\substack{\operatorname{tas} \Lambda_{\theta \rho^{2}} \cap(0, T) \\
\operatorname{esup}}}{\leq} \frac{c}{\theta \rho^{2}} \int_{B_{\rho} \cap \Omega}|u-\varphi|^{2} \mathrm{~d} x \\
& \quad+c \int_{Q_{4 \rho, \theta(4 \rho)^{2}} \cap D}|u-\varphi|^{2} \mathrm{~d} z+\frac{c}{\rho^{p}} \int_{Q_{4 \rho, \theta(4 \rho)^{2}} \cap D}|u-\varphi|^{p} \mathrm{~d} z \\
& \quad f^{p} \mathrm{~d} z .
\end{aligned}
$$

We extend $u(\cdot, t)-\varphi(\cdot, t)$ by zero outside of $\Omega$ and use the same notation for the extension. For a given $t$, we denote

$$
N_{B_{2 \rho}}(u-\varphi)=\left\{x \in \bar{B}_{2 \rho}: u(x, t)-\varphi(x, t)=0\right\} .
$$

We estimate the first term on the right side of (3.16) by using Hölder's inequality and Lemma 3.14. Consequently,

$$
\begin{aligned}
& \frac{c}{\theta \rho^{2}} \int_{Q_{4 \rho, \theta(4 \rho)^{2}} \cap D}|u-\varphi|^{2} \mathrm{~d} z \\
& \leq \frac{c}{\theta \rho^{2}} \int_{\Lambda_{\theta(4 \rho)^{2}} \cap(0, T)} \rho^{n}\left(\frac{1}{\left|B_{4 \rho}\right|} \int_{B_{4 \rho}}|u-\varphi|^{p} \mathrm{~d} x\right)^{2 / p} \mathrm{~d} t \\
& \leq \frac{c \rho^{n}}{\theta \rho^{2}} \int_{\Lambda_{\theta(4 \rho)^{2}} \cap(0, T)}\left(\frac{1}{\operatorname{cap}_{p}\left(N_{B_{2 \rho}}(u-\varphi), B_{4 \rho}\right)} \int_{B_{4 \rho}}|\nabla(u-\varphi)|^{p} \mathrm{~d} x\right)^{2 / p} \mathrm{~d} t
\end{aligned}
$$

Since $\mathbb{R}^{n} \backslash \Omega$ is uniformly $p$-thick and $B_{\frac{4}{3} \rho}\left(x_{0}\right) \backslash \Omega \neq \emptyset$, we conclude by Lemma 3.8 and (3.6) that

$$
\operatorname{cap}_{p}\left(N_{B_{2 \rho}}(u-\varphi), B_{4 \rho}\left(x_{0}\right)\right) \geq \tilde{\mu} \operatorname{cap}_{p}\left(\bar{B}_{2 \rho}\left(x_{0}\right), B_{4 \rho}\left(x_{0}\right)\right)=c \rho^{n-p}
$$

for almost every $t \in[0, T]$. Notice that this estimate still holds true if we redefine $u(\cdot, t)-\varphi(\cdot, t)$ in a set of measure zero in $\Omega$. Next we merge the estimates and obtain

$$
\begin{aligned}
& \frac{c}{\theta \rho^{2}} \int_{Q_{4 \rho, \theta(4 \rho)^{2}} \cap D}|u-\varphi|^{2} \mathrm{~d} z \\
& \leq c \rho^{n+2}\left(\frac{1}{\left|Q_{4 \rho, \theta(4 \rho)^{2}}\right|} \int_{Q_{4 \rho, \theta(4 \rho)^{2}} \cap D}|\nabla(u-\varphi)|^{p} \mathrm{~d} z\right)^{2 / p} .
\end{aligned}
$$

A similar calculation can be repeated for the second term on the right hand side of (3.16), and thus the result follows.

One of the difficulties in proving the main result is the fact that both powers 2 and $p$ play a role in the above inequalities. For example, if we simply divide the term

$$
\frac{c}{\rho^{p}} \int_{Q_{4 \rho, \theta(4 \rho)^{2}} \cap D}|u-\varphi|^{p} \mathrm{~d} z
$$

into two parts as in the quadratic case (see Giaquinta-Struwe [14]), powers do not match. Therefore, we derive a Sobolev-type lemma which takes both powers into account. We again work out the proof in the case $p \leq n$ for simplicity. 
Lemma 3.17. Let $u$ be a global solution with the boundary and initial conditions (3.1). Suppose that $\mathbb{R}^{n} \backslash \Omega$ is uniformly p-thick. Let $\theta>0$ and choose $Q_{\rho, \theta \rho^{2}}=$ $Q_{\rho, \theta \rho^{2}}\left(x_{0}, t_{0}\right) \subset \mathbb{R}^{n+1}$ such that $B_{\frac{4}{3} \rho}\left(x_{0}\right) \backslash \Omega \neq \emptyset$. Then there exist constants $\tilde{q}=\tilde{q}(n, p, \mu)<p$ and $c=c\left(n, p, \mu, \rho_{0}\right)>0$ such that

$$
\begin{aligned}
\frac{1}{\left|Q_{4 \rho, \theta(4 \rho)^{2}}\right|} & \int_{Q_{4 \rho, \theta(4 \rho))^{2} \cap D}|u-\varphi|^{p} \mathrm{~d} z} \\
\leq & \left(\frac{c}{\left|Q_{4 \rho, \theta(4 \rho)^{2}}\right|} \int_{Q_{4 \rho, \theta(4 \rho))^{2}} \cap D}|\nabla(u-\varphi)|^{\tilde{q}} \mathrm{~d} z\right)^{q / \tilde{q}} \\
& \cdot\left(\operatorname{ess~sup}_{t \in \Lambda_{\theta(4 \rho)^{2}} \cap(0, T)} \int_{B_{4 \rho} \cap \Omega}|u-\varphi|^{2} \mathrm{~d} x\right)^{q / n},
\end{aligned}
$$

where $q=p n /(n+2)$.

Proof: The proof is based on Hölder's and Sobolev's inequalities. We set

$$
v(x, t)=|u(x, t)-\varphi(x, t)|,
$$

and employ Hölder's inequality to obtain

$$
\begin{aligned}
\int_{B_{4 \rho} \cap \Omega} v^{p} \mathrm{~d} x & =\int_{B_{4 \rho} \cap \Omega} v^{2 p /(2+n)} v^{p-2 p /(2+n)} \mathrm{d} x \\
& \leq\left(\int_{B_{4 \rho} \cap \Omega} v^{2} \mathrm{~d} x\right)^{q / n}\left(\int_{B_{4 \rho} \cap \Omega} v^{q^{*}} \mathrm{~d} x\right)^{q / q^{*}},
\end{aligned}
$$

where $q^{*}=q n /(n-q)=n p /(n+2-p)$. Observe that $q^{*}$ is well defined provided that $p<n+2$. This condition is satisfied since we assumed that $p \leq n$.

We extend $v(\cdot, t)$ by zero outside of $\Omega$ and use the same notation for the extension. Let $\tilde{q} \geq q$ to be fixed later and set $\tilde{q}^{*}=\tilde{q} n /(n-\tilde{q})$. Furthermore, for a given $t$, denote

$$
N_{B_{2 \rho}}(v)=\left\{x \in \bar{B}_{2 \rho}: v(x, t)=0\right\} .
$$

According to Hölder's inequality and Lemma 3.13, we get

$$
\begin{aligned}
& \left(\int_{B_{4 \rho} \cap \Omega} v^{q^{*}} \mathrm{~d} x\right)^{q / q^{*}} \\
& \quad \leq c \rho^{n q / q^{*}}\left(\frac{1}{\left|B_{4 \rho}\right|} \int_{B_{4 \rho}} v^{\tilde{q}^{*}} \mathrm{~d} x\right)^{q / \tilde{q}^{*}} \\
& \quad \leq c \rho^{n q / q^{*}}\left(\frac{1}{\operatorname{cap}_{\tilde{q}}\left(N_{B_{2 \rho}}(v), B_{4 \rho}\right)} \int_{B_{4 \rho}}|\nabla v|^{\tilde{q}} \mathrm{~d} x\right)^{q / \tilde{q}} .
\end{aligned}
$$

Notice that the assumption $\tilde{q}<p \leq n$ is used here. In the case $\tilde{q}>n$, we should use a different version of Sobolev's inequality.

To continue, we would like to use the uniform capacity density condition, but this is not immediately possible since $\tilde{q}<p$ and since we only assumed that the complement of a domain is uniformly $p$-thick. Nevertheless, Theorem 3.12 asserts 
that the density condition satisfies the self-improving property. This, together with Lemma 3.8 and (3.6), implies

$$
\operatorname{cap}_{\tilde{q}}\left(N_{B_{2 \rho}}(u-\varphi), B_{4 \rho}\right) \geq \tilde{\mu} \operatorname{cap}_{\tilde{q}}\left(\bar{B}_{2 \rho}, B_{4 \rho}\right)=c \rho^{n-\tilde{q}},
$$

for almost every $t$ and for large enough $\tilde{q}<p$. We combine this capacity estimate with (3.18) and conclude that

$$
\left(\int_{B_{4} \cap \Omega} v^{q^{*}} \mathrm{~d} x\right)^{q / q^{*}} \leq c \rho^{n}\left(f_{B_{4 \rho}}|\nabla v|^{\tilde{q}} \mathrm{~d} x\right)^{q / \tilde{q}} .
$$

Collecting the estimates, we arrive at

$$
\begin{aligned}
& \frac{1}{\left|B_{4 \rho}\right|} \int_{B_{4 \rho} \cap D} v^{p} \mathrm{~d} x \\
& \quad \leq c\left(\int_{B_{4 \rho}} v^{2} \mathrm{~d} x\right)^{q / n}\left(\frac{1}{\left|B_{4 \rho}\right|} \int_{B_{4 \rho} \cap D}|\nabla v|^{\tilde{q}} \mathrm{~d} x\right)^{q / \tilde{q}} .
\end{aligned}
$$

The claim follows by integrating this estimate with respect to time and using Hölder's inequality.

\section{Reverse HöLder INEQUALities}

In this section, we derive a reverse Hölder inequality for the gradient of a solution near the lateral boundary and show that this inequality has a self-improving property. We first apply the estimates from the previous section in scaled spacetime cylinders and later use covering arguments to extend the results to general cylinders. The scaling takes both the nonlinearity and the boundary effects into account.

Lemma 4.1 (REVERSE HöLDER). Let $u$ be a global solution with the boundary and initial conditions (3.1). Suppose that $\mathbb{R}^{n} \backslash \Omega$ is uniformly p-thick. Let $\lambda>0$, set $\theta=\lambda^{2-p}$, and choose $Q_{\rho, \theta \rho^{2}}=Q_{\rho, \theta \rho^{2}}\left(x_{0}, t_{0}\right) \subset \mathbb{R}^{n+1}$ such that $B_{\frac{4}{3} \rho}\left(x_{0}\right) \backslash \Omega \neq \emptyset$. Further, choose $M$ such that $\rho<M$ and suppose that there exists a constant $c_{1} \geq 1$ for which

$$
\begin{aligned}
& c_{1}^{-1} \lambda^{p} \leq \frac{1}{\left|Q_{\rho, \theta \rho^{2}}\right|} \int_{Q_{\rho, \theta \rho^{2}} \cap D}\left(|\nabla u|^{p}+f^{p}\right) \mathrm{d} z \\
& \leq \frac{c_{1}}{\left|Q_{20 \rho, \theta(20 \rho)^{2}}\right|} \int_{Q_{20 \rho, \theta(20 \rho)^{2}} \cap D}\left(|\nabla u|^{p}+f^{p}\right) \mathrm{d} z \leq c_{1}^{2} \lambda^{p}, \\
& \frac{1}{\left|Q_{20 \rho, \theta(20 \rho)^{2}}\right|} \int_{Q_{\rho, \theta \rho^{2}} \cap D}|\nabla u|^{p} \mathrm{~d} z \\
& \leq\left(\frac{c}{\left|Q_{4 \rho, \theta(4 \rho)^{2}}\right|} \int_{Q_{4 \rho, \theta(4 \rho)^{2}} \cap D}|\nabla u|^{\tilde{q}} \mathrm{~d} z\right)^{p / \tilde{q}} \\
& +\frac{c}{\left|Q_{4 \rho, \theta(4 \rho)^{2}}\right|} \int_{Q_{4 \rho, \theta(4 \rho)^{2}} \cap D} f^{p} \mathrm{~d} z \text {. }
\end{aligned}
$$


Proof: The idea in the proof is to estimate the terms on the right hand side of Caccioppoli's inequality with the gradient by using the parabolic and capacity versions of Sobolev's inequality. The scaling of the time direction is used in absorbing the additional terms into the left.

Recalling Lemma 3.2, we have

$$
\begin{aligned}
\frac{1}{\left|Q_{\rho, \theta \rho^{2}}\right|} & \int_{Q_{\rho, \theta \rho^{2}} \cap D}\left(|\nabla u|^{p}+f^{p}\right) \mathrm{d} z \\
\leq & \frac{c}{\theta \rho^{2}\left|Q_{4 \rho, \theta(4 \rho)^{2}}\right|} \int_{Q_{4 \rho, \theta(4 \rho)^{2}} \cap D}|u-\varphi|^{2} \mathrm{~d} z \\
& +\frac{c}{\rho^{p}\left|Q_{4 \rho, \theta(4 \rho)^{2}}\right|} \int_{Q_{4 \rho, \theta(4 \rho) 2} \cap D}|u-\varphi|^{p} \mathrm{~d} z \\
& +\frac{c}{\left|Q_{4 \rho, \theta(4 \rho)^{2}}\right|} \int_{Q_{4 \rho, \theta(4 \rho)^{2} \cap D}} f^{p} \mathrm{~d} z .
\end{aligned}
$$

Since $p \geq 2$ and $\theta=\lambda^{2-p}$, we may estimate the first term on the right in terms of the second by using Hölder's and Young's inequalities. We conclude that

$$
\begin{aligned}
& \frac{c}{\theta \rho^{2}\left|Q_{4 \rho, \theta(4 \rho)^{2}}\right|} \int_{Q_{4 \rho, \theta(4 \rho)^{2}} \cap D}|u-\varphi|^{2} \mathrm{~d} z \\
& \leq c \lambda^{p-2}\left(\frac{1}{\rho^{p}\left|Q_{4 \rho, \theta(4 \rho)^{2}}\right|} \int_{Q_{4 \rho, \theta(4 \rho)^{2}} \cap D}|u-\varphi|^{p} \mathrm{~d} z\right)^{2 / p} \\
& \leq \lambda^{p} \varepsilon+\frac{c}{\rho^{p}\left|Q_{4 \rho, \theta(4 \rho)^{2}}\right|} \int_{Q_{4 \rho, \theta(4 \rho)^{2}} \cap D}|u-\varphi|^{p} \mathrm{~d} z,
\end{aligned}
$$

and hence it is enough to estimate the second term on the right hand side of (4.3).

In view of Lemma 3.17, there exists a constant $\tilde{q}<p$ such that

$$
\begin{aligned}
\frac{1}{\left|Q_{4 \rho, \theta(4 \rho)^{2}}\right|} & \int_{Q_{4 \rho, \theta(4 \rho)^{2}} \cap D}|u-\varphi|^{p} \mathrm{~d} z \\
\leq & \left(\frac{c}{\left|Q_{4 \rho, \theta(4 \rho)^{2}}\right|} \int_{Q_{4 \rho, \theta(4 \rho)^{2}} \cap D}|\nabla(u-\varphi)|^{\tilde{q}} \mathrm{~d} z\right)^{q / \tilde{q}} \\
& \cdot\left(\operatorname{essip}_{t \in \Lambda_{\theta(4 \rho)^{2}} \cap(0, T)} \int_{B_{4 \rho}}|u-\varphi|^{2} \mathrm{~d} x\right)^{q / n} \cdot
\end{aligned}
$$

Furthermore, Lemma 3.15 allows us to estimate

$$
\begin{aligned}
& \underset{t \in \Lambda_{\theta(4 \rho)^{2}} \cap(0, T)}{\operatorname{ess} \sup _{B_{4 \rho} \cap \Omega}} \int|u-\varphi|^{2} \mathrm{~d} x \\
& \leq c \rho^{n+2}\left(\frac{1}{\left|Q_{16 \rho, \theta(16 \rho)^{2}}\right|} \int_{Q_{16 \rho, \theta(16 \rho)^{2}} \cap D}|\nabla(u-\varphi)|^{p} \mathrm{~d} z\right)^{2 / p} \\
& \quad+c \int_{Q_{16 \rho, \theta(16 \rho)^{2}} \cap D}|\nabla(u-\varphi)|^{p} \mathrm{~d} z+c \int_{Q_{16 \rho, \theta(16 \rho)^{2}} \cap D} f^{p} \mathrm{~d} z \\
& \leq c \rho^{n+2} \lambda^{2},
\end{aligned}
$$


where we also used assumption (4.2) and the scaling $\theta=\lambda^{2-p}$.

Young's inequality, (4.5) and (4.6) imply

$$
\begin{aligned}
& \frac{c}{\rho^{p}\left|Q_{4 \rho, \theta(4 \rho)^{2}}\right|} \int_{Q_{4 \rho, \theta(4 \rho)^{2}} \cap D}|u-\varphi|^{p} \mathrm{~d} z \\
& \leq \frac{c}{\rho^{p}}\left(\frac{1}{\left|Q_{4 \rho, \theta(4 \rho)^{2}}\right|} \int_{Q_{4 \rho, \theta(4 \rho)^{2}} \cap D}|\nabla(u-\varphi)|^{\tilde{q}} \mathrm{~d} z\right)^{q / \tilde{q}}\left(\rho^{n+2} \lambda^{2}\right)^{q / n} \\
& \leq\left(\frac{c}{\left|Q_{4 \rho, \theta(4 \rho)^{2}}\right|} \int_{Q_{4 \rho, \theta(4 \rho)^{2}} \cap D}|\nabla(u-\varphi)|^{\tilde{q}} \mathrm{~d} z\right)^{p / \tilde{q}}+\varepsilon \lambda^{p},
\end{aligned}
$$

since $\rho^{-p}=\rho^{-(n+2) q / n}$. We combine the previous estimate with (4.3) and (4.4). Thus, we deduce

$$
\begin{aligned}
& \frac{1}{\left|Q_{\rho, \theta \rho^{2}}\right|} \int_{Q_{\rho, \theta \rho^{2}} \cap D}\left(|\nabla u|^{p}+f^{p}\right) \mathrm{d} z \\
& \leq 2 \varepsilon \lambda^{p}+\left(\frac{c}{\left|Q_{4 \rho, \theta(4 \rho)^{2}}\right|} \int_{Q_{4 \rho, \theta(4 \rho)^{2}} \cap D}|\nabla(u-\varphi)|^{\tilde{q}} \mathrm{~d} z\right)^{p / \tilde{q}} \\
& \quad+\frac{c}{\left|Q_{4 \rho, \theta(4 \rho)^{2}}\right|} \int_{Q_{4 \rho, \theta(4 \rho) 2} \cap D} f^{p} \mathrm{~d} z .
\end{aligned}
$$

By assumption (4.2), we have

$$
c_{1}^{-1} \lambda^{p} \leq \frac{1}{\left|Q_{\rho, \theta \rho^{2}}\right|} \int_{Q_{\rho, \theta \rho^{2}} \cap D}\left(|\nabla u|^{p}+f^{p}\right) \mathrm{d} z
$$

and, as a consequence, we can choose $\varepsilon>0$ small enough to absorb $2 \varepsilon \lambda^{p}$ into the left hand side. Finally, since (4.2) implies

$$
\begin{aligned}
\frac{1}{\left|Q_{20 \rho, \theta(20 \rho)^{2}}\right|} & \int_{Q_{20 \rho, \theta(20 \rho)^{2}} \cap D}|\nabla u|^{p} \mathrm{~d} z \\
& \leq \frac{c}{\left|Q_{\rho, \theta \rho^{2}}\right|} \int_{Q_{\rho, \theta \rho^{2}} \cap D}\left(|\nabla u|^{p}+f^{p}\right) \mathrm{d} z
\end{aligned}
$$

we have proven the claim.

Next we prove that the reverse Hölder inequality has a self-improving property. In the case $p=2$, we can use the well-known Giaquinta-Modica lemma, which can be found from Giaquinta-Modica [13] or from Giaquinta [12]. See also Gehring [11], Stredulinsky [27] and Giaquinta-Struwe [14]. Since now $p \geq 2$, we follow a different strategy: We split the space-time domain into scaled cylinders so that the reverse Hölder inequality holds in each of them.

We say that $Q_{4 R,(4 R)^{2}}\left(x_{0}, t_{0}\right)$ intersects the lateral boundary if

$$
Q_{4 R,(4 R)^{2}}\left(x_{0}, t_{0}\right) \cap(\partial \Omega \times[0, T]) \neq \emptyset,
$$

and that $Q_{4 R,(4 R)^{2}}\left(x_{0}, t_{0}\right)$ intersects the initial boundary if

$$
Q_{4 R,(4 R)^{2}}\left(x_{0}, t_{0}\right) \cap(\Omega \times\{0\}) \neq \emptyset .
$$


Furthermore, we denote

$$
\begin{aligned}
& \tilde{V}_{\delta}^{p}(0, T ; \Omega) \\
& =\left\{\varphi \in W^{1, p /(p-1)+\delta}\left(0, T ; L^{p /(p-1)+\delta}(\Omega)\right) \cap L^{p+\delta}\left(0, T ; W^{1, p+\delta}(\Omega)\right):\right. \\
& \left.\varphi \in C\left([0, T] ; L^{2}(\Omega)\right), \varphi(\cdot, 0) \in W^{1, q+\delta}(\Omega)\right\},
\end{aligned}
$$

where $\delta>0$ and $q=p n /(n+2)$.

Theorem 4.7. Let $u$ be a global solution to (2.2) satisfying the boundary and initial conditions (3.1) for a boundary function

$$
\varphi \in \tilde{V}_{\delta}^{p}(0, T ; \Omega),
$$

where $\delta>0$. Suppose that $\mathbb{R}^{n} \backslash \Omega$ is uniformly $p$-thick and that $R<M$ for some $M>0$. Choose $Q_{R, R^{2}}=Q_{R, R^{2}}\left(x_{0}, t_{0}\right) \subset \mathbb{R}^{n+1}$ such that $Q_{4 R,(4 R)^{2}}$ intersects the lateral and initial boundaries. Then there exist constants $\varepsilon_{0}=$ $\varepsilon_{0}\left(n, p, M, \delta, \rho_{0}, \mu, \alpha, \beta\right)>0$ and $c>0$ with the same dependencies such that for all $0 \leq \varepsilon<\varepsilon_{0}$, we have

$$
\begin{aligned}
\left(\frac{1}{\left|Q_{R, R^{2}}\right|}\right. & \left.\int_{Q_{R, R^{2} \cap D}}|\nabla u|^{p+\varepsilon} \mathrm{d} z\right)^{1 /(p+\varepsilon)} \\
\leq & \left(\frac{c}{\left|B_{4 R}\right|} \int_{B_{4 R} \cap \Omega} \tilde{f}^{q+\varepsilon} \mathrm{d} x\right)^{1 /(q+\varepsilon)} \\
& +\left(\frac{c}{\left|Q_{4 R,(4 R)^{2}}\right|} \int_{Q_{4 R,(4 R)^{2} \cap D}}\left(|\nabla u|^{p}+f^{p+\varepsilon}\right) \mathrm{d} z\right)^{1 /(p+\varepsilon)} \\
& +\left(\frac{c}{\left|Q_{4 R,(4 R)^{2}}\right|} \int_{Q_{4 R,(4 R)^{2} \cap D}}\left(|\nabla u|^{p}+f^{p}\right) \mathrm{d} z\right)^{\sigma},
\end{aligned}
$$

where $\sigma=(2+\varepsilon) /(2(p+\varepsilon)), q=p n /(n+2), \tilde{f}=|\nabla \varphi(x, 0)|$, and $f=\left(|\nabla \varphi|^{p}+\right.$ $\left.\left|\varphi^{\prime}\right|^{p /(p-1)}\right)^{1 / p}$.

Proof: The proof consists of several steps. First, we cover the space-time cylinder with smaller Whitney-type cylinders. By using the Whitney cylinders, we are able to derive estimates with constants independent of the location. Then we divide the space-time cylinder into a good and a bad set. In the good set, the function $|\nabla u|^{p}$ is in control by definition, and in the bad set, we can estimate the average of the gradient by using the reverse Hölder inequality. The CalderónZygmund decomposition is usually applied for this but here we use a different strategy which seems to work better in the parabolic setting with general growth conditions. Finally, we obtain the higher integrability by using Fubini's theorem.

We denote $Q_{0}=Q_{4 R,(4 R)^{2}}\left(z_{0}\right)=Q_{4 R,(4 R)^{2}}\left(x_{0}, t_{0}\right)$ and divide $Q_{0}$ into the Whitney-type cylinders

$$
Q_{i}=Q_{r_{i}, r_{i}^{2}}\left(y_{i}, \tau_{i}\right), i=1,2, \ldots,
$$

where $r_{i}$ is comparable to the parabolic distance of $Q_{i}$ to the $\partial Q_{0}$ (see, for example, page 15 of [26]). Parabolic distance is defined to be

$$
\operatorname{dist}_{p}(E, F)=\inf \left\{|x-\bar{x}|+|t-\bar{t}|^{1 / 2}:(x, t) \in E,(\bar{x}, \bar{t}) \in F\right\} .
$$


In addition, cylinders $Q_{i}$ are of bounded overlap, meaning that every $z$ belongs at most to a fixed finite number of cylinders, and

$$
Q_{5 r_{i},\left(5 r_{i}\right)^{2}} \subset Q_{0} .
$$

The next step is to divide $Q_{0}$ into a good and a bad set. We aim to choose the scaling $\lambda>0$ so that condition (4.2) holds in the cylinders having a center point in the bad set. To this end, set

$$
\lambda_{0}^{\prime}=\left(\frac{1}{\left|Q_{0}\right|} \int_{Q_{0} \cap D}\left(|\nabla u|^{p}+f^{p}\right) \mathrm{d} z\right)^{1 / 2},
$$

and choose $\lambda$ such that

$$
\lambda>\max \left(\lambda_{0}^{\prime}, 1\right)=\lambda_{0}
$$

For $(x, t) \in Q_{0} \cap D$, we define

$$
h(x, t)=\frac{1}{c_{2}\left|Q_{0}\right|^{1 / 2}} \min \left\{\left|Q_{i}\right|^{1 / 2}:(x, t) \in Q_{i}\right\}|\nabla u(x, t)|,
$$

where $c_{2} \geq 1$ is fixed later. Further, choose $(\tilde{x}, \tilde{t}) \in D$ such that

$$
h(\tilde{x}, \tilde{t})>\lambda
$$

and fix $Q_{i}$ for which $(\tilde{x}, \tilde{t}) \in Q_{i} \cap D$. We define

$$
\alpha=\alpha(\tilde{x}, \tilde{t})=\frac{\left|Q_{0}\right|}{\left|Q_{i}\right|},
$$

and

$$
\theta=\lambda^{2-p} \alpha^{1-p / 2}
$$

If $(\tilde{x}, \tilde{t})$ belongs to many Whitney cylinders, any of them will do.

Next we show that the second inequality in condition (4.2) is valid due to the definition of $\lambda$. For $Q_{r, \theta r^{2}}=Q_{r, \theta r^{2}}(\tilde{x}, \tilde{t}), r_{i} / 20 \leq r \leq r_{i}$, we obtain

$$
\begin{aligned}
\frac{1}{\left|Q_{r, \theta r^{2}}\right|} & \int_{Q_{r, \theta r^{2} \cap D}}\left(|\nabla u|^{p}+f^{p}\right) \mathrm{d} z \\
& \leq \frac{c\left|Q_{0}\right|}{\left|Q_{i}\right| \theta} \frac{1}{\left|Q_{0}\right|} \int_{Q_{0} \cap D}\left(|\nabla u|^{p}+f^{p}\right) \mathrm{d} z \\
& \leq c_{2}{ }^{p} \alpha^{p / 2} \lambda^{p}
\end{aligned}
$$

where $c_{2}$ is chosen to be large enough. The first inequality in (4.2) will be valid for small cylinders due to Lebesgue's differentiation theorem. We arrive at

$$
\lim _{r^{\prime} \rightarrow 0} \frac{1}{\left|Q_{r^{\prime}, \theta r^{\prime 2}}\right|} \int_{Q_{r^{\prime}, \theta r^{\prime 2}}(\tilde{x}, \tilde{t})}\left(|\nabla u|^{p}+f^{p}\right) \mathrm{d} z>c_{2}{ }^{p} \alpha^{p / 2} \lambda^{p},
$$

which holds for almost every $(\tilde{x}, \tilde{t}) \in Q_{i} \cap D$ such that $h(\tilde{x}, \tilde{t})>\lambda$. An appropriate version of Lebesgue's differentiation theorem is proven in Zygmund [28].

Observe that the integral above is continuous with respect to $r$. Furthermore, the integral is less than or equal to $c_{2}{ }^{p} \alpha^{p / 2} \lambda^{p}$ for all $r, r_{i} / 20 \leq r \leq r_{i}$, and greater than $c_{2}^{p} \alpha^{p / 2} \lambda^{p}$ for $r$ small enough. Thus, there exists $\rho_{1}, 0<\rho_{1} \leq r_{i} / 20$, such that the integral equals $c_{2}^{p} \alpha^{p / 2} \lambda^{p}$ if $r=\rho_{1}$. Moreover, for all larger values of $r$, the 
integral is less than or equal to $c_{2}^{p} \alpha^{p / 2} \lambda^{p}$. Consequently, there exists a constant $c \geq 1$, independent of the location, such that

$$
\begin{aligned}
& c^{-1} \alpha^{p / 2} \lambda^{p} \\
& \leq \frac{1}{\left|Q_{\rho_{1}, \theta \rho_{1}^{2}}\right|} \int_{Q_{\rho_{1}, \theta \rho_{1}^{2}} \cap D}\left(|\nabla u|^{p}+f^{p}\right) \mathrm{d} z \\
& \leq \frac{c}{\left|Q_{20 \rho_{1}, \theta\left(20 \rho_{1}\right)^{2}}\right|} \int_{Q_{20 \rho_{1}, \theta\left(20 \rho_{1}\right)^{2}} \cap D}\left(|\nabla u|^{p}+f^{p}\right) \mathrm{d} z \leq c^{2} \alpha^{p / 2} \lambda^{p} .
\end{aligned}
$$

A similar reasoning implies that there exists $\rho_{2}<\rho_{1}, 0<\rho_{2} \leq r_{i} / 20$, such that

$$
\begin{aligned}
c^{-1} \alpha^{p / 2} \lambda^{p} & \leq \frac{1}{\left|Q_{\rho_{2}, \theta \rho_{2}^{2}}\right|} \int_{Q_{\rho_{2}, \theta \rho_{2}^{2}} \cap D}|\nabla u|^{p} \mathrm{~d} z \\
& \leq \frac{c}{\left|Q_{20 \rho_{2}, \theta\left(20 \rho_{2}\right)^{2}}\right|} \int_{Q_{20 \rho_{2}, \theta\left(20 \rho_{2}\right)^{2}} \cap D}|\nabla u|^{p} \mathrm{~d} z \leq c^{2} \alpha^{p / 2} \lambda^{p} .
\end{aligned}
$$

At this point, we remark that $\alpha, \lambda>1$, and, therefore, $\theta<1$ as well as $Q_{20 \rho_{1}, \theta\left(20 \rho_{1}\right)^{2}} \subset Q_{0}$.

If $\lambda$ is replaced by $\alpha^{1 / 2} \lambda$, then (4.8) shows that condition (4.2) in Lemma 4.1 holds with $\rho_{1}$ whenever $h(\tilde{x}, \tilde{t})>\lambda$ and, further, $\theta \rho^{2}=\left(\alpha^{1 / 2} \lambda\right)^{2-p} \rho^{2}$. If $B_{\frac{4}{3} \rho_{1}}(\tilde{x}) \backslash$ $\Omega \neq \emptyset$, then Lemma 4.1 implies

$$
\begin{aligned}
\frac{1}{\left|Q_{\rho_{1}, \theta \rho_{1}^{2}}\right|} \int_{Q_{\rho_{1}, \theta \rho_{1}^{2}} \cap D}|\nabla u|^{p} \mathrm{~d} z \\
\leq\left(\frac{c}{\left|Q_{4 \rho_{1}, \theta\left(4 \rho_{1}\right)^{2}}\right|} \int_{\left.Q_{4 \rho_{1}, \theta\left(4 \rho_{1}\right)^{2} \cap D}|\nabla u|^{\tilde{q}} \mathrm{~d} z\right)^{p / \tilde{q}}} f^{p} \mathrm{~d} z,\right. \\
\quad+\frac{c}{\left|Q_{4 \rho_{1}, \theta\left(4 \rho_{1}\right)^{2}}\right|} \int_{Q_{4 \rho_{1}, \theta\left(4 \rho_{1}\right)^{2} \cap D}}
\end{aligned}
$$

for some $\tilde{q}<p$.

Assume then that $B_{\frac{4}{3} \rho_{2}}(\tilde{x}) \backslash \Omega=\emptyset$ and denote $q=p n /(n+2)$. If $Q_{\frac{7}{6} \rho_{2}, \theta\left(\frac{7}{6} \rho_{2}\right)^{2}}$ does not intersect the initial boundary, we obtain a local result

$$
\begin{aligned}
\frac{1}{\left|Q_{\rho_{2}, \theta \rho_{2}^{2}}\right|} & \int_{Q_{\rho_{2}, \theta \rho_{2}^{2}} \cap D}|\nabla u|^{p} \mathrm{~d} z \\
& \leq c\left(\left|Q_{\frac{7}{6} \rho_{2}, \theta\left(\frac{7}{6} \rho_{2}\right)^{2}}\right|^{-1} \int_{Q_{\frac{7}{6} \rho_{2}, \theta\left(\frac{7}{6} \rho_{2}\right)^{2}} \cap D}|\nabla u|^{q} \mathrm{~d} z\right)^{p / q} \\
& \leq\left(\frac{c}{\left|Q_{4 \rho_{2}, \theta\left(4 \rho_{2}\right)^{2}}\right|} \int_{Q_{4 \rho_{2}, \theta\left(4 \rho_{2}\right)^{2}} \cap D}|\nabla u|^{\tilde{q}} \mathrm{~d} z\right)^{p / \tilde{q}},
\end{aligned}
$$

where Hölder's inequality was applied in the last step. For the proof of the local result, we refer to Lemma 3.4 in Kinnunen-Lewis [19]. 
If $B_{\frac{4}{3} \rho_{2}}(\tilde{x}) \backslash \Omega=\emptyset$ and if $Q_{\frac{7}{6} \rho_{2}, \theta\left(\frac{7}{6} \rho_{2}\right)^{2}}$ intersects the initial boundary, then we obtain an initial boundary estimate

$$
\begin{aligned}
\frac{1}{\left|Q_{\rho_{2}, \theta \rho_{2}^{2}}\right|} & \int_{Q_{\rho_{2}, \theta \rho 2} \cap D}|\nabla u|^{p} \mathrm{~d} z \\
\leq & \left(\frac{c}{\left|Q_{4 \rho_{2}, \theta\left(4 \rho_{2}\right)^{2}}\right|} \int_{\left.Q_{4 \rho_{2}, \theta\left(4 \rho_{2}\right)^{2} \cap D}|\nabla u|^{\tilde{q}} \mathrm{~d} z\right)^{p / \tilde{q}}}\right. \\
& +\left(\frac{c}{\left|B_{4 \rho_{2}}\right|} \int_{B_{4 \rho_{2}} \cap \Omega} \tilde{f}^{q} \mathrm{~d} x\right)^{p / q},
\end{aligned}
$$

where $\tilde{f}=|\nabla \varphi(x, 0)|$. Due to our assumptions on the initial values, the last term is well defined. Estimate (4.12) can be derived in much the same way as the local result in [19] since (4.9) is available. In order to avoid lenghty and partly repetitious calculations, we omit the further details.

Let us now return to the case $B_{\frac{4}{3} \rho_{1}}(\tilde{x}) \backslash \Omega \neq \emptyset$. From (4.8), we obtain

$$
\begin{aligned}
& c^{-1} \lambda^{p} \\
& \leq \frac{1}{\left|Q_{\rho_{1}, \theta \rho_{1}^{2}}\right|} \int_{Q_{\rho_{1}, \theta \rho_{1}^{2}} \cap D}\left(h^{p}+\alpha^{-p / 2} f^{p}\right) \mathrm{d} z \\
& \leq \frac{c}{\left|Q_{20 \rho_{1}, \theta\left(20 \rho_{1}\right)^{2}}\right|} \int_{Q_{20 \rho_{1}, \theta\left(20 \rho_{1}\right)^{2} \cap D}}\left(h^{p}+\alpha^{-p / 2} f^{p}\right) \mathrm{d} z \leq c^{2} \lambda^{p},
\end{aligned}
$$

since the volumes of all the Whitney cylinders intersecting $Q_{20 \rho_{1}, \theta\left(20 \rho_{1}\right)^{2}}$ are comparable. In view of (4.10) and (4.13), we have

$$
\begin{aligned}
\frac{1}{\left|Q_{20 \rho_{1}, \theta\left(20 \rho_{1}\right)^{2}}\right|} & \int_{Q_{20 \rho_{1}, \theta\left(20 \rho_{1}\right)^{2} \cap D}} h^{p}+\alpha^{-p / 2} f^{p} \mathrm{~d} z \\
\leq & \left(\frac{c}{\left|Q_{4 \rho_{1}, \theta\left(4 \rho_{1}\right)^{2}}\right|} \int_{Q_{4 \rho_{1}, \theta\left(4 \rho_{1}\right)^{2}} \cap D} h^{\tilde{q} \mathrm{~d} z)^{p / \tilde{q}}}\right. \\
& +\frac{c}{\left|Q_{4 \rho_{1}, \theta\left(4 \rho_{1}\right)^{2}}\right|} \int_{Q_{4 \rho_{1}, \theta\left(4 \rho_{1}\right)^{2}} \cap D} \alpha^{-p / 2} f^{p} \mathrm{~d} z .
\end{aligned}
$$

Next we decompose $Q_{0}$ into level sets. We define

$$
G(\lambda)=\left\{(x, t) \in Q_{0} \cap D: h(x, t)>\lambda\right\}
$$

and

$$
\tilde{G}(\lambda)=\left\{(x, t) \in Q_{0} \cap D: f(x, t)>\lambda\right\} .
$$

Since $h(x, t)>\lambda$ in $G(\lambda)$, we can later use the previous estimates in $G(\lambda)$.

Observe that

$$
h(x, t) \leq \eta \lambda \quad \text { whenever } \quad(x, t) \in\left(Q_{4 \rho_{1}, \theta\left(4 \rho_{1}\right)^{2}} \cap D\right) \backslash G(\eta \lambda),
$$

and

$$
f(x, t) \leq \eta \lambda \quad \text { whenever } \quad(x, t) \in\left(Q_{4 \rho_{1}, \theta\left(4 \rho_{1}\right)^{2}} \cap D\right) \backslash \tilde{G}(\eta \lambda) .
$$

Furthermore, since

$$
\alpha^{-p / 2}=\left(\left|Q_{i}\right| /\left|Q_{0}\right|\right)^{p / 2} \leq 1
$$


we obtain by (4.14) that

$$
\begin{aligned}
& \frac{1}{\left|Q_{20 \rho_{1}, \theta\left(20 \rho_{1}\right)^{2}}\right|} \int_{Q_{20 \rho_{1}, \theta\left(20 \rho_{1}\right)^{2} \cap D}}\left(h^{p}+\alpha^{-p / 2} f^{p}\right) \mathrm{d} z \\
& \leq c \eta^{p} \lambda^{p}+\left(\frac{c}{\left|Q_{4 \rho_{1}, \theta\left(4 \rho_{1}\right)^{2}}\right|} \int_{Q_{4 \rho_{1}, \theta\left(4 \rho_{1}\right)^{2}} \cap G(\eta \lambda)} h^{\tilde{q}} \mathrm{~d} z\right)^{p / \tilde{q}} \\
& \quad+\frac{c}{\left|Q_{4 \rho_{1}, \theta\left(4 \rho_{1}\right)^{2}}\right|} \int_{Q_{4 \rho_{1}, \theta\left(4 \rho_{1}\right)^{2}} \cap \tilde{G}(\eta \lambda)} f^{p} \mathrm{~d} z .
\end{aligned}
$$

By Hölder's inequality and (4.13), there exists a constant $c \geq 1$ such that

$$
\left(\frac{1}{\left|Q_{4 \rho_{1}, \theta\left(4 \rho_{1}\right)^{2}}\right|} \int_{Q_{4 \rho_{1}, \theta\left(4 \rho_{1}\right)^{2}} \cap D} h^{\tilde{q}} \mathrm{~d} z\right)^{(p-\tilde{q}) / \tilde{q}} \leq c \lambda^{p-\tilde{q}} .
$$

To continue, we choose $\eta>0$ small enough to absorb the first term on the right hand side of (4.15) into the left. This is possible due to (4.13). We combine the result with (4.16), multiply by $\left|Q_{20 \rho_{1}, \theta\left(20 \rho_{1}\right)^{2}}\right|$ and get

$$
\begin{aligned}
\int_{Q_{20 \rho_{1}, \theta\left(20 \rho_{1}\right)^{2} \cap D}} h^{p} \mathrm{~d} z \leq & c \lambda^{p-\tilde{q}} \int_{Q_{4 \rho_{1}, \theta\left(4 \rho_{1}\right)^{2}} \cap G(\eta \lambda)} h^{\tilde{q} \mathrm{~d} z} \\
& +c \int_{Q_{\mu_{1}, \theta\left(4 \rho_{1}\right)^{2}} \cap \tilde{G}(\eta \lambda)} f^{p} \mathrm{~d} z .
\end{aligned}
$$

If $B_{\frac{4}{3} \rho_{2}}(\tilde{x}) \backslash \Omega=\emptyset$ and if $Q_{\frac{7}{6} \rho_{2}, \theta\left(\frac{7}{6} \rho_{2}\right)^{2}}$ does not intersect the initial boundary, then we obtain a local version of the above estimate by using (4.9) and (4.11). Consequently,

$$
\begin{aligned}
\int_{Q_{20 \rho_{2}, \theta\left(20 \rho_{2}\right)^{2}} \cap D} h^{p} \mathrm{~d} z & \leq c \lambda^{p-\tilde{q}} \int_{Q_{\frac{7}{6} \rho_{2}, \theta\left(\frac{7}{6} \rho_{2}\right)^{2}} \cap G(\eta \lambda)} h^{\tilde{q} \mathrm{~d} z} \\
& \leq c \lambda^{p-\tilde{q}} \int_{Q_{4 \rho_{2}, \theta\left(4 \rho_{2}\right)^{2}} \cap G(\eta \lambda)} h^{\tilde{q}} \mathrm{~d} z .
\end{aligned}
$$

Finally, if $B_{\frac{4}{3} \rho_{2}}(\tilde{x}) \backslash \Omega=\emptyset$ and if $Q_{\frac{7}{6} \rho_{2}, \theta\left(\frac{7}{6} \rho_{2}\right)^{2}}$ intersects the initial boundary, then we obtain an initial boundary version by using (4.9) and (4.12). Since $\left|Q_{20 \rho_{2}, \theta\left(20 \rho_{2}\right)^{2}}\right| \leq c\left|B_{4 \rho_{2}}\right|^{p / q}$, we deduce

$$
\begin{aligned}
\int_{Q_{20 \rho_{2}, \theta\left(20 \rho_{2}\right)^{2}} \cap D} h^{p} \mathrm{~d} z \leq & c \lambda^{p-\tilde{q}} \int_{Q_{4 \rho_{2}, \theta\left(4 \rho_{2}\right)^{2}} \cap G(\eta \lambda)} h^{\tilde{q}} \mathrm{~d} z \\
& +\left(c \int_{B_{4 \rho_{2}} \cap \bar{G}(\eta \lambda)} \tilde{f}^{q} \mathrm{~d} x\right)^{p / q}
\end{aligned}
$$

where

$$
\bar{G}(\eta \lambda)=\left\{x \in B_{4 R}\left(x_{0}\right) \cap \Omega: \tilde{f}(x)>\eta \lambda\right\} .
$$

Since $\rho_{2}<\rho_{1}$, either $B_{\frac{4}{3} \rho_{2}}(\tilde{x}) \backslash \Omega=\emptyset$ and (4.9) holds or $B_{\frac{4}{3} \rho_{1}}(\tilde{x}) \backslash \Omega \neq \emptyset$ and (4.8) holds. Thus one of the above estimates is always available.

As a next step, we use a covering argument to extend the estimates to the whole of $G(\lambda)$. By Vitali's covering theorem, we have a disjoint set of cylinders

$$
\left\{Q_{4 \rho_{i}^{\prime}, \theta\left(4 \rho_{i}^{\prime}\right)}\left(\tilde{z}_{i}\right)\right\}_{i=1}^{\infty}, \quad \tilde{z}_{i} \in G(\lambda), \quad \tilde{z}_{i}=\left(\tilde{x}_{i}, \tilde{t}_{i}\right)
$$


such that almost everywhere

$$
G(\lambda) \subset \bigcup_{i=1}^{\infty} Q_{20 \rho_{i}^{\prime}, \theta\left(20 \rho_{i}^{\prime}\right)^{2}}\left(\tilde{z}_{i}\right) \subset Q_{0},
$$

and either (4.17), (4.18), or (4.19) holds in each of the cylinders. Then we sum over $i$ and obtain

$$
\begin{aligned}
& \int_{G(\lambda)} h^{p} \mathrm{~d} z \leq \sum_{i=1}^{\infty} \int_{Q_{20 \rho_{i}^{\prime}, \theta\left(20 \rho_{i}^{\prime}\right)^{2}\left(\tilde{z}_{i}\right) \cap D} h^{p} \mathrm{~d} z} h^{\leq} \sum_{i=1}^{\infty}\left(\lambda^{p-\tilde{q}} \int_{\left.Q_{4 \rho_{i}^{\prime}, \theta\left(4 \rho_{i}^{\prime}\right)^{\prime}\left(\tilde{z}_{i}\right) \cap G(\eta \lambda)} \mathrm{d} z+b_{i}\right)}\right. \\
& \leq c \lambda^{p-\tilde{q}} \int_{G(\eta \lambda)} h^{\tilde{q}} \mathrm{~d} z+c \int_{\tilde{G}(\eta \lambda)} f^{p} \mathrm{~d} z+c\left(\int_{\bar{G}(\eta \lambda)} \tilde{f}^{q} \mathrm{~d} x\right)^{p / q},
\end{aligned}
$$

where $b_{i}$ is either the lateral boundary term, initial boundary term, or zero depending on the corresponding estimate. When summing over the initial boundary terms, we used the fact $p / q>1$.

The higher integrability result is now a consequence of (4.21) and Fubini's theorem. To see this, we integrate over $G\left(\lambda_{0}\right)$ and use (4.21) together with Fubini's theorem. Thus,

$$
\begin{aligned}
& \int_{G\left(\lambda_{0}\right)} h^{p+\varepsilon} \mathrm{d} z=\int_{G\left(\lambda_{0}\right)}\left(\int_{\lambda_{0}}^{h} \varepsilon \lambda^{\varepsilon-1} \mathrm{~d} \lambda+\left(\lambda_{0}\right)^{\varepsilon}\right) h^{p} \mathrm{~d} z \\
& =\varepsilon \int_{\lambda_{0}}^{\infty} \lambda^{\varepsilon-1} \int_{G(\lambda)} h^{p} \mathrm{~d} z \mathrm{~d} \lambda+\left(\lambda_{0}\right)^{\varepsilon} \int_{G\left(\lambda_{0}\right)} h^{p} \mathrm{~d} z \\
& \leq c \int_{\lambda_{0}}^{\infty}\left(\varepsilon \lambda^{\varepsilon-1+p-\tilde{q}} \int_{G(\eta \lambda)} h^{\tilde{q}} \mathrm{~d} z+\varepsilon \lambda^{\varepsilon-1} \int_{\tilde{G}(\eta \lambda)} f^{p} \mathrm{~d} z\right. \\
& \left.+\varepsilon \lambda^{\varepsilon-1}\left(\int_{\bar{G}(\eta \lambda)} \tilde{f}^{q} \mathrm{~d} x\right)^{p / q}\right) \mathrm{d} \lambda+\left(\lambda_{0}\right)^{\varepsilon} \int_{G\left(\lambda_{0}\right)} h^{p} \mathrm{~d} z .
\end{aligned}
$$

We estimate the right hand side in three parts. First, by Fubini's theorem, we see that

$$
\begin{aligned}
& \varepsilon \int_{\lambda_{0}}^{\infty} \lambda^{\varepsilon-1+p-\tilde{q}} \int_{G(\eta \lambda)} h^{\tilde{q}} \mathrm{~d} z \mathrm{~d} \lambda+\left(\lambda_{0}\right)^{\varepsilon} \int_{G\left(\lambda_{0}\right)} h^{p} \mathrm{~d} z \\
& =c \varepsilon \int_{G\left(\eta \lambda_{0}\right)} \int_{\lambda_{0}}^{h / \eta} \lambda^{\varepsilon-1+p-\tilde{q}} h^{\tilde{q}} \mathrm{~d} \lambda \mathrm{d} z+\left(\lambda_{0}\right)^{\varepsilon} \int_{G\left(\lambda_{0}\right)} h^{p} \mathrm{~d} z \\
& \leq \frac{c \varepsilon}{\varepsilon+p-\tilde{q}} \int_{G\left(\eta \lambda_{0}\right)}\left(h^{\varepsilon+p} \eta^{\tilde{q}-p-\varepsilon}-\left(\lambda_{0}\right)^{\varepsilon+p-\tilde{q}} h^{\tilde{q}}\right) \mathrm{d} z+\left(\lambda_{0}\right)^{\varepsilon} \int_{G\left(\lambda_{0}\right)} h^{p} \mathrm{~d} z .
\end{aligned}
$$


Since the second term in the first integral is negative, it follows that

$$
\begin{aligned}
& \frac{c \varepsilon}{\varepsilon+p-\tilde{q}} \int_{G\left(\eta \lambda_{0}\right)}\left(h^{\varepsilon+p} \eta^{\tilde{q}-p-\varepsilon}-\lambda_{0}{ }^{\varepsilon+p-\tilde{q}} h^{\tilde{q}}\right) \mathrm{d} z \\
& \leq \frac{c \varepsilon}{\varepsilon+p-\tilde{q}}\left(\int_{G\left(\lambda_{0}\right)} h^{\varepsilon+p} \eta^{\tilde{q}-p-\varepsilon} \mathrm{d} z+\left(\lambda_{0}\right)^{\varepsilon} \int_{G\left(\eta \lambda_{0}\right) \backslash G\left(\lambda_{0}\right)} h^{p} \eta^{\tilde{q}-p-\varepsilon} \mathrm{d} z\right) \\
& \leq \frac{c \varepsilon}{\varepsilon+p-\tilde{q}} \int_{G\left(\lambda_{0}\right)} h^{\varepsilon+p} \mathrm{~d} z+c\left(\lambda_{0}\right)^{\varepsilon} \int_{G\left(\eta \lambda_{0}\right)} h^{p} \mathrm{~d} z,
\end{aligned}
$$

where we also used the fact that $\lambda_{0} \geq h$ in $G\left(\eta \lambda_{0}\right) \backslash G\left(\lambda_{0}\right)$. We end up with

$$
\begin{aligned}
& \varepsilon \int_{\lambda_{0}}^{\infty} \lambda^{\varepsilon-1+p-\tilde{q}} \int_{G(\eta \lambda)} h^{\tilde{q}} \mathrm{~d} z \mathrm{~d} \lambda+\left(\lambda_{0}\right)^{\varepsilon} \int_{G\left(\lambda_{0}\right)} h^{p} \mathrm{~d} z \\
& \quad \leq \frac{c \varepsilon}{\varepsilon+p-\tilde{q}} \int_{G\left(\lambda_{0}\right)} h^{\varepsilon+p} \mathrm{~d} z+c\left(\lambda_{0}\right)^{\varepsilon} \int_{G\left(\eta \lambda_{0}\right)} h^{p} \mathrm{~d} z .
\end{aligned}
$$

Let us now estimate the lateral boundary term in (4.22). We utilize Fubini's theorem and obtain

$$
\begin{aligned}
\varepsilon \int_{\lambda_{0}}^{\infty} \lambda^{\varepsilon-1} \int_{\tilde{G}(\eta \lambda)} f^{p} \mathrm{~d} z \mathrm{~d} \lambda & =\int_{\tilde{G}\left(\eta \lambda_{0}\right)}\left((f / \eta)^{\varepsilon}-\left(\lambda_{0}\right)^{\varepsilon}\right) f^{p} \mathrm{~d} z \\
& \leq c \int_{\tilde{G}\left(\eta \lambda_{0}\right)} f^{\varepsilon+p} \mathrm{~d} z .
\end{aligned}
$$

To estimate the initial boundary term in (4.22), we divide the term into two parts as

$$
\begin{aligned}
& \varepsilon \int_{\lambda_{0}}^{\infty} \lambda^{\varepsilon-1}\left(\int_{\bar{G}(\eta \lambda)} \tilde{f}^{q} \mathrm{~d} x\right)^{p / q} \mathrm{~d} \lambda \\
& \quad \leq\left(\int_{\bar{G}\left(\eta \lambda_{0}\right)} \tilde{f}^{q} \mathrm{~d} x\right)^{p / q-1} \int_{\lambda_{0}}^{\infty} \varepsilon \lambda^{\varepsilon-1} \int_{\bar{G}(\eta \lambda)} \tilde{f}^{q} \mathrm{~d} x \mathrm{~d} \lambda
\end{aligned}
$$

Now we can apply Fubini's theorem to estimate the second part. The first part can be estimated by using Hölder's inequality. It follows that

$$
\begin{aligned}
& \varepsilon \int_{\lambda_{0}}^{\infty} \lambda^{\varepsilon-1}\left(\int_{\bar{G}(\eta \lambda)} \tilde{f}^{q} \mathrm{~d} x\right)^{p / q} \mathrm{~d} \lambda \\
& \leq\left(\int_{\bar{G}\left(\eta \lambda_{0}\right)} \tilde{f}^{q} \mathrm{~d} x\right)^{p / q-1} \int_{\bar{G}\left(\eta \lambda_{0}\right)} \int_{\lambda_{0}}^{\tilde{f} / \eta} \varepsilon \lambda^{\varepsilon-1} \tilde{f}^{q} \mathrm{~d} \lambda \mathrm{d} x \\
& \leq c R^{2 \varepsilon /(q+\varepsilon)}\left(\int_{\bar{G}\left(\eta \lambda_{0}\right)} \tilde{f}^{q+\varepsilon} \mathrm{d} x\right)^{(p+\varepsilon) /(q+\varepsilon)}
\end{aligned}
$$

Now we are ready to collect the estimates. We combine (4.23), (4.24), and (4.25) with (4.22). Then we choose $\varepsilon>0$ small enough to absorb the term containing 
$h^{p+\varepsilon}$ into the left hand side and get

$$
\begin{aligned}
\int_{G\left(\lambda_{0}\right)} h^{p+\varepsilon} \mathrm{d} z \leq & c\left(\lambda_{0}\right)^{\varepsilon} \int_{G\left(\eta \lambda_{0}\right)} h^{p} \mathrm{~d} z+c \int_{\tilde{G}\left(\eta \lambda_{0}\right)} f^{p+\varepsilon} \mathrm{d} z \\
& +c R^{2 \varepsilon /(q+\varepsilon)}\left(\int_{\bar{G}\left(\eta \lambda_{0}\right)} \tilde{f}^{q+\varepsilon} \mathrm{d} x\right)^{(p+\varepsilon) /(q+\varepsilon)}
\end{aligned} .
$$

Notice that if the term we would like to absorb is infinite, then we can replace $h$ by $h_{k}=\min \{h, k\}, k>\lambda_{0}$. Indeed, estimate (4.21) continues to hold in the form

$$
\begin{aligned}
\int_{\left\{h_{k}>\lambda\right\}} h_{k}^{p-\tilde{q}} \mathrm{~d} \mu \leq & c \lambda^{p-\tilde{q}} \int_{\left\{h_{k}>\eta \lambda\right\}} \mathrm{d} \mu \\
& +c \int_{\tilde{G}(\eta \lambda)} f^{p} \mathrm{~d} z+c\left(\int_{\bar{G}(\eta \lambda)} \tilde{f}^{q} \mathrm{~d} x\right)^{p / q},
\end{aligned}
$$

where $\mathrm{d} \mu=h^{\tilde{q}} \mathrm{~d} z$. Then we use this estimate in the calculations starting from (4.22) and have

$$
\begin{aligned}
\int_{\left\{h_{k}>\lambda_{0}\right\}} h_{k}^{p-\tilde{q}+\varepsilon} \mathrm{d} \mu \leq & \frac{c \varepsilon}{\varepsilon+p-\tilde{q}} \int_{\left\{h_{k}>\lambda_{0}\right\}} h_{k}^{p-\tilde{q}+\varepsilon} \mathrm{d} \mu \\
& +c\left(\lambda_{0}\right)^{\varepsilon} \int_{\left\{h_{k}>\eta \lambda_{0}\right\}} h_{k}^{p-\tilde{q}} \mathrm{~d} \mu+c \int_{\tilde{G}\left(\eta \lambda_{0}\right)} f^{p+\varepsilon} \mathrm{d} z \\
& +c R^{2 \varepsilon /(q+\varepsilon)}\left(\int_{\bar{G}\left(\eta \lambda_{0}\right)} \tilde{f}^{q+\varepsilon} \mathrm{d} x\right)^{(p+\varepsilon) /(q+\varepsilon)}
\end{aligned}
$$

As a result, we can absorb the first term on the right hand side into the left and then employ Lebesgue's monotone convergence theorem to let $k \rightarrow \infty$. Thus, we obtain (4.26).

Since $h \leq \lambda_{0}$ in $\left(Q_{0} \cap D\right) \backslash G\left(\lambda_{0}\right)$, estimate (4.26) extends to the whole of $Q_{R, R^{2}} \cap D$. Indeed,

$$
\begin{aligned}
\int_{Q_{R, R^{2}} \cap D} h^{p+\varepsilon} \mathrm{d} z \leq & \left(\lambda_{0}\right)^{\varepsilon} \int_{\left(Q_{0} \cap D\right) \backslash G\left(\lambda_{0}\right)} h^{p} \mathrm{~d} z+\int_{G\left(\lambda_{0}\right)} h^{p+\varepsilon} \mathrm{d} z \\
\leq & c\left(\lambda_{0}\right)^{\varepsilon} \int_{Q_{0} \cap D} h^{p} \mathrm{~d} z+c \int_{Q_{0} \cap D} f^{p+\varepsilon} \mathrm{d} z \\
& +c R^{2 \varepsilon /(q+\varepsilon)}\left(\int_{B_{0} \cap \Omega} \tilde{f}^{q+\varepsilon} \mathrm{d} x\right)^{(p+\varepsilon) /(q+\varepsilon)}
\end{aligned}
$$

We divide the estimate by $\left|Q_{0}\right|$ and apply the definition of $h(z)$. Since $Q_{R, R^{2}}$ lies far away from the boundary of $Q_{0}=Q_{4 R,(4 R)^{2}}$, there exists $c>0$, independent of $R$, such that

$$
\min \left\{\left|Q_{i}\right|^{1 / 2}:(x, t) \in Q_{i}\right\} /\left|Q_{0}\right|^{1 / 2}>c
$$

for every $(x, t) \in Q_{R, R^{2}} \cap D$. On the right hand side, we estimate

$$
\min \left\{\left|Q_{i}\right|^{1 / 2}:(x, t) \in Q_{i}\right\} /\left|Q_{0}\right|^{1 / 2} \leq 1,
$$


and, consequently,

$$
\begin{aligned}
& \frac{1}{\left|Q_{0}\right|} \int_{Q_{R, R^{2}} \cap D}|\nabla u|^{p+\varepsilon} \mathrm{d} z \leq \frac{c\left(\lambda_{0}\right)^{\varepsilon}}{\left|Q_{0}\right|} \int_{Q_{0} \cap D}|\nabla u|^{p} \mathrm{~d} z \\
& +\frac{c}{\left|Q_{0}\right|} \int_{Q_{0} \cap D} f^{p+\varepsilon} \mathrm{d} z+\left(\frac{c}{\left|B_{0}\right|} \int_{B_{0} \cap \Omega} \tilde{f}^{q+\varepsilon} \mathrm{d} x\right)^{(p+\varepsilon) /(q+\varepsilon)} .
\end{aligned}
$$

Next we take the cut-off level into account. Remember that either

$$
\lambda_{0}=1 \quad \text { or } \quad \lambda_{0}=\lambda_{0}^{\prime} .
$$

The first case is clear. Moreover, if $\lambda_{0}=\lambda_{0}^{\prime}$, then Young's inequality and the definition of $\lambda_{0}^{\prime}$ leads to

$$
\begin{aligned}
\frac{1}{\left|Q_{R, R^{2}}\right|} \int_{Q_{R, R^{2}} \cap D}|\nabla u|^{p+\varepsilon} \mathrm{d} z \leq\left(\frac{c}{\left|Q_{0}\right|} \int_{Q_{0} \cap D}|\nabla u|^{p} \mathrm{~d} z\right)^{(\varepsilon+2) / 2} \\
+\left(\frac{c}{\left|Q_{0}\right|} \int_{Q_{0} \cap D} f^{p} \mathrm{~d} z\right)^{(\varepsilon+2) / 2}+\frac{c}{\left|Q_{0}\right|} \int_{Q_{0} \cap D} f^{p+\varepsilon} \mathrm{d} z \\
+\left(\frac{c}{\left|B_{0}\right|} \int_{B_{0} \cap \Omega} \tilde{f}^{q+\varepsilon} \mathrm{d} x\right)^{(p+\varepsilon) /(q+\varepsilon)} \cdot
\end{aligned}
$$

This finishes the proof.

\section{ACKNOWLEDGEMENTS}

I wish to express my gratitude to Professor Juha Kinnunen for useful discussions. For financial support, I am indebted to the Magnus Ehrnrooth Foundation and the Finnish Academy of Science and Letters, the Vilho, Yrjö and Kalle Väisälä Foundation.

\section{REFERENCES}

[1] E. Acerbi and G. Mingione. Gradient estimates for a class of parabolic systems. Duke Math. J., 136(2):285-320, 2007.

[2] A. Ancona. On strong barriers and an inequality of Hardy for domains in $\mathbf{R}^{n}$. J. London Math. Soc. (2), 34(2):274-290, 1986.

[3] A. A. Arkhipova. $L^{p}$-estimates for the gradients of solutions of initial boundary value problems to quasilinear parabolic systems (Russian). St.Petersburg State Univ., Problems of Math. Analysis, 13:5-18, 1992.

[4] A. A. Arkhipova. Reverse Hölder inequalities with boundary integrals and $L^{p}$-estimates for solutions of nonlinear elliptic and parabolic boundary-value problems. In Nonlinear evolution equations, volume 164 of Amer. Math. Soc. Transl. Ser. 2, pages 15-42. Amer. Math. Soc., Providence, RI, 1995.

[5] B. V. Bojarski. Generalized solutions of a system of differential equations of first order and of elliptic type with discontinuous coefficients (Russian). Mat. Sb. N.S., 43(85):451-503, 1957.

[6] E. DiBenedetto. Degenerate parabolic equations. Universitext. Springer-Verlag, New York, 1993.

[7] F. Duzaar and G. Mingione. The $p$-harmonic approximation and the regularity of $p$-harmonic maps. Calc. Var. Partial Differential Equations, 20(3):235-256, 2004.

[8] F. Duzaar and G. Mingione. Second order parabolic systems, optimal regularity, and singular sets of solutions. Ann. Inst. H. Poincaré Anal. Non Linéaire, 22(6):705-751, 2005.

[9] A. Elcrat and N. G. Meyers. Some results on regularity for solutions of non-linear elliptic systems and quasi-regular functions. Duke Math. J., 42:121-136, 1975.

[10] L. C. Evans and R. F. Gariepy. Measure theory and fine properties of functions. Studies in Advanced Mathematics. CRC Press, Boca Raton, FL, 1992. 
[11] F. W. Gehring. The $L^{p}$-integrability of the partial derivatives of a quasiconformal mapping. Acta Math., 130:265-277, 1973.

[12] M. Giaquinta. Multiple integrals in the calculus of variations and nonlinear elliptic systems, volume 105 of Annals of Mathematics Studies. Princeton University Press, Princeton, NJ, 1983.

[13] M. Giaquinta and G. Modica. Regularity results for some classes of higher order nonlinear elliptic systems. J. Reine Angew. Math., 311/312:145-169, 1979.

[14] M. Giaquinta and M. Struwe. On the partial regularity of weak solutions of nonlinear parabolic systems. Math. Z., 179(4):437-451, 1982.

[15] S. Granlund. An $L^{p}$-estimate for the gradient of extremals. Math. Scand., 50(1):66-72, 1982.

[16] L. I. Hedberg. Spectral synthesis in Sobolev spaces, and uniqueness of solutions of the Dirichlet problem. Acta Math., 147(3-4):237-264, 1981.

[17] J. Heinonen, T. Kilpeläinen, and O. Martio. Nonlinear potential theory of degenerate elliptic equations. Oxford Mathematical Monographs. Oxford University Press, New York, 1993.

[18] T. Kilpeläinen and P. Koskela. Global integrability of the gradients of solutions to partial differential equations. Nonlinear Anal., 23(7):899-909, 1994.

[19] J. Kinnunen and J. L. Lewis. Higher integrability for parabolic systems of $p$-Laplacian type. Duke Math. J., 102(2):253-271, 2000.

[20] J. Kristensen and G. Mingione. The singular set of minima of integral functionals. Arch. Ration. Mech. Anal., 180(3):331-398, 2006.

[21] J. L. Lewis. Uniformly fat sets. Trans. Amer. Math. Soc., 308(1):177-196, 1988.

[22] J. Malý and W. P. Ziemer. Fine regularity of solutions of elliptic partial differential equations, volume 51 of Mathematical Surveys and Monographs. American Mathematical Society, Providence, RI, 1997.

[23] V. G. Maz'ja. Sobolev spaces. Springer Series in Soviet Mathematics. Springer-Verlag, Berlin, 1985.

[24] P. Mikkonen. On the Wolff potential and quasilinear elliptic equations involving measures. Ann. Acad. Sci. Fenn. Math. Diss., 104:1-71, 1996.

[25] M. Parviainen. Global higher integrability for parabolic quasiminimizers in nonsmooth domains. Calc. Var. Partial Differential Equations, 31(1):75-98, 2008.

[26] E. M. Stein. Harmonic analysis: real-variable methods, orthogonality, and oscillatory integrals, volume 43 of Princeton Mathematical Series. Princeton University Press, Princeton, NJ, 1993.

[27] E. W. Stredulinsky. Higher integrability from reverse Hölder inequalities. Indiana Univ. Math. J., 29(3):407-413, 1980.

[28] A. Zygmund. On the differentiability of multiple integrals. Fund. Math., 23:143-149, 1934. 